NASA/TM-1998-112223

$$
\begin{aligned}
& 1 N-08 \\
& 081469
\end{aligned}
$$

\title{
Optimization of Supersonic Transport Trajectories
}

Mark D. Ardema, Robert Windhorst, and James Phillips 
Since its founding, NASA has been dedicated to the advancement of aeronautics and space science. The NASA Scientific and Technical Information (STI) Program Office plays a key part in helping NASA maintain this important role.

The NASA STI Program Office is operated by Langley Research Center, the Lead Center for NASA's scientific and technical information. The NASA STI Program Office provides access to the NASA STI Database, the largest collection of aeronautical and space science STI in the world. The Program Office is also NASA's institutional mechanism for disseminating the results of its research and development activities. These results are published by NASA in the NASA STI Report Series, which includes the following report types:

- TECHNICAL PUBLICATION. Reports of completed research or a major significant phase of research that present the results of NASA programs and include extensive data or theoretical analysis. Includes compilations of significant scientific and technical data and information deemed to be of continuing reference value. NASA's counterpart of peer-reviewed formal professional papers but has less stringent limitations on manuscript length and extent of graphic presentations.

- TECHNICAL MEMORANDUM. Scientific and technical findings that are preliminary or of specialized interest, e.g., quick release reports, working papers, and bibliographies that contain minimal annotation. Does not contain extensive analysis.

- CONTRACTOR REPORT. Scientific and technical findings by NASA-sponsored contractors and grantees.
- CONFERENCE PUBLICATION. Collected papers from scientific and technical conferences, symposia, seminars, or other meetings sponsored or cosponsored by NASA.

- SPECIAL PUBLICATION. Scientific, technical, or historical information from NASA programs, projects, and missions, often concerned with subjects having substantial public interest.

- TECHNICAL TRANSLATION. Englishlanguage translations of foreign scientific and technical material pertinent to NASA's mission.

Specialized services that complement the STI Program Office's diverse offerings include creating custom thesauri, building customized databases, organizing and publishing research results ... even providing videos.

For more information about the NASA STI

Program Office, see the following:

- Access the NASA STI Program Home Page at http://www.sti.nasa.gov

- E-mail your question via the Internet to help@sti.nasa.gov

- Fax your question to the NASA Access Help Desk at (301) 621-0134

- Telephone the NASA Access Help Desk at (301) 621-0390

- Write to: NASA Access Help Desk NASA Center for AeroSpace Information 800 Elkridge Landing Road Linthicum Heights, MD 21090-2934 
NASA/TM-1998-112223

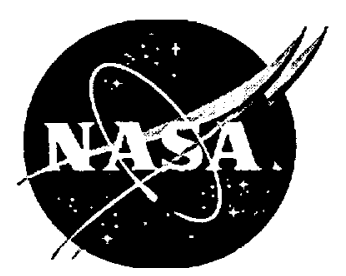

\section{Optimization of Supersonic Transport Trajectories}

Mark D. Ardema and Robert Windhorst

Santa Clara University, Santa Clara, Califormia

James Phillips

Ames Research Center, Moffett Field, California

National Aeronautics and

Space Administration

Ames Research Center

Moffett Field, California 93035-1000 
Available from:

NASA Center for AeroSpace Information 800 Elkridge Landing Road

Linthicum Heights, MD 21090-2934

Price Code: A 17
National Technical Information Service 5285 Port Royal Road Springfield, VA 22161 Price Code: A10 


\section{CONTENTS}

$\begin{array}{ll}\text { SUMMARY } & 1\end{array}$

INTRODUCTION

DYNAMIC MODELING 2

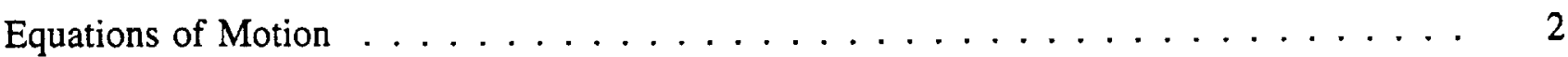

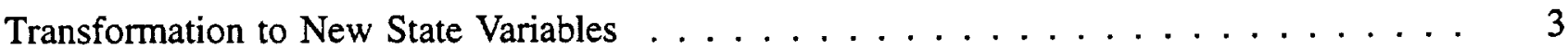

OPTIMAL CONTROL AND SINGULAR PERTURBATIONS 9

The Maximum Principle . . . . . . . . . . . . . . . . . . . . 9

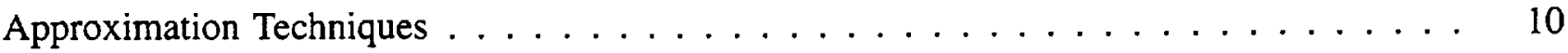

Singular Perturbations and Time Scaling .................... 11

GUIDANCE LAW DEVELOPMENT 15

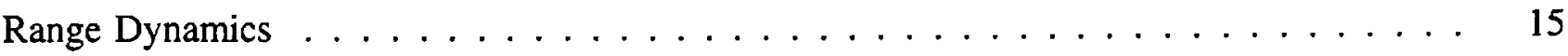

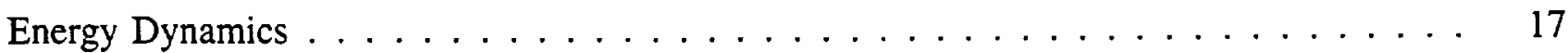

Fast States Dynamics . . . . . . . . . . . . . . . . . . . . . 19

$\begin{array}{lr}\text { NUMERICAL EXAMPLE } & 23\end{array}$

$\begin{array}{lr}\text { CONCLUDING REMARKS } & 26\end{array}$

APPENDIX A - NOMENCLATURE 27

APPENDIX B - NUMERICAL INTEGRATION OF STATE EQUATIONS 28

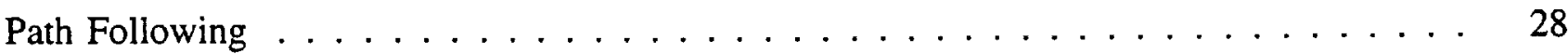

Constant Normal Load Factor $(N)$ Paths . . . . . . . . . . . . . . . . . . . . 29

APPENDIX C - NECESSARY CONDITIONS FOR FAST DYNAMICS 32

$\begin{array}{ll}\text { REFERENCES } & 38\end{array}$

$\begin{array}{lr}\text { TABLE } & 41\end{array}$

$\begin{array}{lr}\text { FIGURES } & 41\end{array}$ 
• 


\section{SUMMARY}

This paper develops a near-optimal guidance law for generating minimum fuel, time, or cost fixedrange trajectories for supersonic transport aircraft. The approach uses a choice of new state variables along with singular perturbation techniques to time-scale decouple the dynamic equations into multiple equations of single order (second order for the fast dynamics). Application of the maximum principle to each of the decoupled equations, as opposed to application to the original coupled equations, avoids the two point boundary value problem and transforms the problem from one of a functional optimization to one of multiple function optimizations. It is shown that such an approach produces well known aircraft performance results such as minimizing the Brequet factor for minimum fuel consumption and the energy climb path. Furthermore, the new state variables produce a consistent calculation of flight path angle along the trajectory, eliminating one of the deficiencies in the traditional energy state approximation. In addition, jumps in the energy climb path are smoothed out by integration of the original dynamic equations at constant load factor. Numerical results performed for a supersonic transport design show that a pushover dive followed by a pullout at nominal load factors are sufficient maneuvers to smooth the jump.

\section{INTRODUCTION}

The purpose of this work is to develop and implement a near-optimal guidance law for use in an aircraft synthesis computer code, such as the ACSYNT code ${ }^{1}$ developed at NASA Ames Research Center. Of primary interest is the optimization of supersonic transport trajectories. ACSYNT, like other such codes, models all aspects (aerodynamics, propulsion, structures, weights, etc.) of an aircraft design to produce consistent performance estimates. It is capable of computing "closed" vehicles, that is, designs that meet mission requirements, by iteratively adjusting vehicle parameters. It is also capable of optimizing design parameters, again by iteratively cycling through the code.

A key element of any vehicle synthesis code is the trajectory calculation. Because the trajectory routine is exercised repeatedly in the course of a design study, it must be efficient, robust, and userfriendly. "Exact" trajectory optimization, relying on optimal control theory, requires iterative solution of an unstable two-point boundary-value problem (2PBVP), and therefore is not suitable for this application. Thus simplifying approximations are required.

It has long been known that if there is but one state equation, then the functional optimization problem (2PBVP) reduces to a function one ${ }^{2,3,4}$. A natural and well-established way to effect the required order reduction is to time-scale the system state equations and then apply singular perturbation techniques (see for example refs. 2-9). If each state variable is put on its own time-scale then the problem is thereby reduced to a sequence of function optimizations.

The main problem with completely time-scaling the aircraft dynamics is that speed and altitude are not time-scale separable. This is usually resolved by replacing the speed by the total mechanical energy as a state variable (see for example refs. 2-12), and we adopt this approach here. In addition, another new state variable is introduced to replace the altitude, one which removes the inconsistency in flight path angle $11,13,14$ that occurs in the energy dynamics with the usual formulation. This does not 
directly impact the energy dynamics solution but increases the accuracy of the altitude/fiight path angle dynamics solution.

The energy-state approximation (neglecting all dynamics except the energy dynamics) has been applied with success to a wide variety of aircraft, including high performance supersonic aircraft and launch vehicles. It is perhaps best suited, however, to transport aircraft because the benign maneuvers of these vehicles make the assumptions involved in the energy-state approximation (ESA) less questionable. The ESA has been applied most thoroughly to subsonic transport aircraft by Erzberger ${ }^{15-17}$. The results were so satisfactory that the resulting algorithms currently are being used for on-board guidance in commercial transports.

Applying the ESA to supersonic transports introduces some new features. First, these aircraft have higher speeds and usually longer ranges than do subsonic aircraft. More importantly, due to the rise in drag near transonic speeds, they typically have an instantaneous altitude change in their energy-climb paths. These altitude jumps have been investigated by various means in references $12,18-22$. In this paper, we use the approach of references 12 and 22 to address this problem.

Finally, some numerical results are presented to demonstrate the utility of the method.

\section{DYNAMIC MODELING}

\section{Equations of Motion}

The equations of vehicle motion in ACSYNT are:

$$
\begin{aligned}
& \dot{m}=-\beta=-\pi T_{m} C \\
& \dot{x}=v \cos \gamma \\
& \dot{v}=\frac{T \cos \alpha-D-m g \sin \gamma}{m} \\
& \dot{h}=v \sin \gamma \\
& \dot{\gamma}=\frac{T \sin \alpha+L-m g \cos \gamma}{m v}
\end{aligned}
$$

These equations assume no winds, thrust direction fixed with respect to the aircraft body, and a non-rotating flat earth. A linear throttle is not assumed; that is, specific fuel consumption, $C$, varies with thrust. The symbols used here and throughout this report are defined in Appendix A.

To simplify the terms, define the tangential and normal load factors as

$$
\begin{aligned}
& F=\frac{(T \cos \alpha-D)}{m g} \\
& N=\frac{(T \sin \alpha+L)}{m g}
\end{aligned}
$$


Then equations (1) become

$$
\begin{aligned}
& \dot{m}=-\beta \\
& \dot{x}=v \cos \gamma \\
& \dot{v}=g(F-\sin \gamma) \\
& \dot{h}=v \sin \gamma \\
& \dot{\gamma}=\frac{g}{v}(N-\cos \gamma)
\end{aligned}
$$

In ACSYNT, as in many other vehicle synthesis codes, equations (3) are numerically integrated, with the $\dot{\gamma}$ term set to zero, for a specified set of ordered pairs of altitude and Mach number (or speed). The methods used for this integration are given in Appendix $\mathrm{B}$. The $(h, M)$ points needed for the integration may come from any number of sources, for example a constant dynamic pressure (constant equivalent airspeed) path or an external trajectory optimization. It is our purpose to develop an algorithm that generates these points near-optimally for some prescribed cost functional "on the fly", that is, as the trajectory integration proceeds.

\section{Transformation to New State Variables}

Experience has shown that the state variables in equations (3) have a natural time-scale separation for most vehicles and most missions, except that $h$ and $v$ are on almost the same time scale. To time scale separate these variables, we seek a new variable, $E(h, v)$, to replace $v$, such that the state equation for $E$ is independent of $?^{11.13 .14}$. Taking the time derivative of $E$ and using equations (3):

$$
\dot{E}=E_{h} \dot{h}+E_{v} \dot{v}=E_{h} v \sin \gamma+E_{v} g(F-\sin \gamma)
$$

Throughout, the following notation will be used: If $Q$ is any function of $h$ and $v$, then

$$
Q_{h}=\left.\frac{\partial Q}{\partial h}\right|_{v}, \quad Q_{v}=\left.\frac{\partial Q}{\partial v}\right|_{h}
$$

If $\dot{E}$ is to be independent of $\gamma$, from equation (4):

$$
E_{h} v-E_{v} g=0
$$

The solution of this equation is

$$
E=h+\frac{1}{2 g} v^{2}
$$


or any once-differentiable function of this. From equation (6) we see that $E$ is just the total mechanical energy of the aircraft per unit weight. Substituting $E$ for $v$ as a state variable gives

$$
\begin{aligned}
& \dot{m}=-\beta \\
& \dot{x}=v \cos \gamma \\
& \dot{E}=v F \\
& \dot{h}=v \sin \gamma \\
& \dot{\gamma}=\frac{g}{v}(N-\cos \gamma)
\end{aligned}
$$

Numerous analyses have shown that there is a strong time-scale separation between $E$ and $h$ (see for example refs. 23,24$)$. In equations (8), $v$ is to be regarded as a function of $E$ and $h$, as given by equations (7):

$$
v=\sqrt{2 g(E-h)}
$$

The product $v F$ in the third of equations (7) is usually called the specific excess power.

Equations (7), along with a suitably defined cost functional, define an optimal control problem in the five states $m, x, E, h$, and $\gamma$, with control $\alpha$ (and possibly throttle if it is allowed to vary). The boundary conditions on these states are

$$
\begin{array}{ll}
m(0)=m_{0} & m\left(t_{f}\right) \text { free } \\
x(0)=0 & x\left(t_{f}\right)=R \\
E(0)=E_{0} & E\left(t_{f}\right)=E_{f} \\
h(0)=h_{0} & h\left(t_{f}\right)=h_{f} \\
\gamma(0)=\gamma_{0} & \gamma\left(t_{f}\right)=\gamma_{f}
\end{array}
$$

where $t_{f}$ is free.

The following constraints are placed on the trajectory:

1. Maximum dynamic pressure, $q(h, v) \leq q_{m}$

2. Maximum Mach number, $M(h, v) \leq M_{m}$

3. Maximum lift coefficient, $c_{L}(h, v) \leq c_{L_{m}}$

4. Minimum terrain limit, $h \geq h_{m}$

5. Maximum loft ceiling (locus of flight conditions for which $F=0$ for maximum throttle and $\gamma=0)$ 
All of these constraints may be written as functions of $h$ and $M$ or of $h$ and $E$; when drawn in the $(h, M)$ plane (fig. 1) they define the flight envelope. In the context of equations (7) they are state inequality constraints of the form:

$$
s_{i}(h, v) \leq 0 ; \quad i=1, \cdot \cdot, 5
$$

Optimal control problems with state inequality constraints are a difficult class of problems for several reasons ${ }^{25,26}$.

The complete time-scale decoupling of equations (7) will be formulated later. At present, for the sake of dynamic modeling, it is instructive to consider the energy-state approximation (ESA) associated with equations (7); it is:

$$
\begin{aligned}
& m=\text { const } \\
& x=\text { const } \\
& \dot{E}=v F \\
& 0=v \sin \gamma \\
& 0=\frac{g}{v}(N-\cos \gamma)
\end{aligned}
$$

The fourth of these implies that $\gamma=0$ and the fifth then gives $\alpha$ as a function of $h$ and $E$. The problem thus reduces to a single state equation with $h$ (and possibly throttle) as control and $E$ as state. The solution, for a suitable cost functional, may be put into the form (see later)

$$
f(h, v)=0
$$

This will be called the energy-climb path, or ECP. This may be either one of the constraints equations (10) or an interior extremal. One of the main advantages of the ESA is that it converts the state variable inequality constraints, equations (10), into state-dependent control inequality constraints, a much simpler situation from an optimal control point of view.

Since equation (12) generally gives $\dot{h} \neq 0, \gamma$ will not be zero on the ECP, giving a contradiction. What is needed is a new variable that is constant along the ECP. An obvious choice is $f$ itself since by equation (12) $\dot{f}=0$ along the $\mathrm{ECP}^{11,13,14}$. Since $d f=f_{h} d h+f_{v} d v=0$ we have

$$
\frac{d v}{d h}=-\frac{f_{h}}{f_{v}}
$$

But from equations (3)

$$
\frac{d v}{d h}=\frac{g(F-\sin \gamma)}{v \sin \gamma}
$$


so that

$$
\gamma=\sin ^{-1}\left(\frac{F}{1-\frac{v}{g} \frac{f_{h}}{f_{v}}}\right)
$$

This is the consistent value of $\gamma$ along the ECP. Also, from equations (3)

$$
\begin{gathered}
\dot{f}=f_{h} \dot{h}+f_{v} \dot{v} \\
\dot{f}=f_{h} v \sin \gamma+f_{v} g(F-\sin \gamma)
\end{gathered}
$$

Note that the choice of variable $f$ actually depends on the nature of the ECP and may vary along the trajectory. The equations of motion in the new variables are now:

$$
\begin{aligned}
\dot{m} & =-\beta \\
\dot{x} & =v \cos \gamma \\
\dot{E} & =v F \\
\dot{f} & =f_{h} v \sin \gamma+f_{v} g(F-\sin \gamma) \\
\dot{\gamma} & =\frac{g}{v}(N-\cos \gamma)
\end{aligned}
$$

These equations are entirely equivalent to equations (1).

Some examples of the function $f$ will now be given.

1. ECP on a terrain limit:

$$
\begin{gathered}
f(h, v)=h-h_{m}=0 \\
f_{h}=1, \quad f_{v}=0
\end{gathered}
$$

From equations (15) and (16):

$$
\begin{aligned}
& \gamma=0 \\
& \dot{f}=v \sin \gamma
\end{aligned}
$$

2. ECP on a dynamic pressure limit:

$$
f(h, v)=\frac{1}{2} \rho(h) v^{2}-q_{m}=0
$$




$$
\begin{gathered}
f_{h}=\frac{1}{2} \rho_{h} v^{2}, \quad f_{v}=\rho v \\
\gamma=\sin ^{-1}\left(\frac{F}{1-\frac{\rho_{h} v^{2}}{2 g \rho}}\right) \\
\dot{f}=\frac{1}{2} \rho_{h} v^{3} \sin \gamma+\rho v g(F-\sin \gamma)
\end{gathered}
$$

3. ECP an interior unbounded extremal. In this case $(\partial(v F) / \partial h)_{E}=0$ so that:

$$
\begin{gathered}
f(h, v)=F+v F_{v}-\frac{v^{2}}{g} F_{h}=0 \\
f_{h}=F_{h}+v F_{v h}-\frac{v^{2}}{g} F_{h h} \\
f_{v}=2 F_{v}+v F_{v v}-\frac{2 v}{g} F_{h}-\frac{v^{2}}{g} F_{h v} \\
\gamma=\sin ^{-1}\left(\frac{F}{1-\frac{v\left(g F_{h}+g v F_{v h}-v^{2} F_{h h}\right)}{g\left(2 g F_{v}+g v F_{v v}-2 v F_{h}-v^{2} F_{v h}\right.}}\right) \\
\dot{f}=v\left(F_{h}+v F_{v h}-\frac{v^{2}}{g} F_{h h}\right) \sin \gamma+g\left(2 F_{v}+v F_{v v}-\frac{2 v}{g} F_{h}-\frac{v^{2}}{g} F_{h v}\right)(F-\sin \gamma)
\end{gathered}
$$

Since this latter case involves second derivatives of $F$, usually a severe problem when dealing with numerically defined functions as in the case here, for this case it is probably preferable to compute $\gamma$ along the ECP directly from equation (14)

$$
\gamma=\sin ^{-1}\left(\frac{F}{1+\frac{v}{g} \frac{d v}{d h}}\right)
$$

where $d v / d h$ is evaluated numerically along the ESA solution. These examples show that the usual choice of variable in the ESA, $h$, is only valid when the ECP is on a terrain limit.

Now consider the ESA associated with equations (17):

$$
\begin{aligned}
& m=\text { const. } \\
& x=\text { const. } \\
& \dot{E}=v F \\
& 0=f_{h} v \sin \gamma+f_{v} g(F-\sin \gamma) \\
& 0=\frac{g}{v}(N-\cos \gamma)
\end{aligned}
$$


The fourth and fifth of these are to be solved for $\alpha$ and $\gamma$ as functions of $E$ and $f$. Direct elimination of $\gamma$ gives

$$
0=f_{h} v\left(\sqrt{1-N^{2}}\right)+f_{v} g\left(F-\sqrt{1-N^{2}}\right)
$$

and thus the restriction $-1 \leq N \leq 1$ must be imposed. Since we will need to consider cases $N>1$ later on, this restriction is unacceptable. The problem is resolved by making the small $\gamma$ assumption ( $\sin \gamma=\gamma, \cos \gamma=1$ ), a very good approximation for transport aircraft whose flight path angles are at most a few degrees. Finally then, the equations of motion we shall be dealing with are

$$
\begin{aligned}
& \dot{m}=-\beta \\
& \dot{x}=v \\
& \dot{E}=v F \\
& \dot{f}=f_{h} v \gamma+f_{v} g(F-\gamma) \\
& \dot{\gamma}=\frac{g}{v}(N-1)
\end{aligned}
$$

with boundary conditions

$$
\begin{array}{ll}
m(0)=m_{0} & m\left(t_{f}\right) \text { free } \\
x(0)=0 & x\left(t_{f}\right)=R \\
E(0)=E_{0} & E\left(t_{f}\right)=E_{f} \\
f(0)=f_{0} & f\left(t_{f}\right)=f_{f} \\
\gamma(0)=\gamma_{0} & \gamma\left(t_{f}\right)=\gamma_{f}
\end{array}
$$

where the boundary conditions on $E$ and $f$ are determined by the boundary conditions on $h$ and $v$, and $t_{f}$ is free. 


\section{OPTIMAL CONTROL AND SINGULAR PERTURBATIONS}

\section{The Maximum Principle}

All of the equations of motion of the previous section (see for example equations (19)) are of state variable form:

$$
\underline{\dot{x}}=\underline{f}(\underline{x}, \underline{u})
$$

where $\underline{x} \in \mathbb{R}^{n}$ is the state and $\underline{u} \in U \subset \mathbb{R}^{m}$ is the control. Suitable boundary conditions on the state vector components are prescribed (see for example eqs. (20)). It is desired to find the components of $\underline{u}$ along the trajectory such that a cost functional

$$
J=\int_{0}^{t_{f}} \phi(\underline{x}, \underline{u}) d t
$$

is minimized. It is assumed that the final time, $t_{f}$, is free. Extensions of this basic problem such as for terminal cost or fixed final time are easily made, but are not of interest here.

Theorem (the maximum principle) ${ }^{25,27,28}$ : Introduce the variational Hamiltonian function

$$
H=\lambda_{0} \phi+\sum_{i=1}^{n} \lambda_{i} f_{i}
$$

where the components of the adjoint vector, $\underline{\lambda}$, satisfy the differential equations

$$
\dot{\lambda}_{i}=-\frac{\partial H}{\partial x_{i}} ; \quad i=1, \cdot \cdot n
$$

Then, if $\underline{u}$ is an optimal control, there exists a nontrivial solution of equations (24) such that

(a) $\underline{u}=\arg \max H$

$\underline{u} \in U$

(b) $H=0$

(c) Transversality conditions ("natural" boundary conditions on the $\lambda_{i}$ ) hold

(d) $\lambda_{0}=$ const. $\leq 0$

In the sequel it is assumed that $\lambda_{0}=0$ does not lead to a solution and therefore we may take $\lambda_{0}=-1$ (this scales the adjoint variables $\lambda_{i}$ ).

The maximum principle gives the control as a function of time or of the state variables. When this function is substituted into equations (21) and (24), the result is a $2 n$ dimension $2 P B V P$ in the states and adjoints. Exactly $n$ boundary conditions are provided at $t=0$ and the other $n$ at $t=t_{f}$ (due to 
the transversality conditions). Further, the equations are unstable in the sense that if they are linearized about a nominal trajectory, one-half of the system matrix eigenvalues will have positive real parts and the other negative (unless some are zero). Although many approaches have been developed to solve this class of problem, they are all computationally expensive (requiring repetitive solution of the equations), non-robust (due to the instability), and not user-friendly (requiring extensive input by experts). Thus they are unsuitable for use in a vehicle synthesis code and approximations must be developed for this purpose.

\section{Approximation Techniques}

Our basic approach is to reduce the complexity of the trajectory optimization problem by seeking means of reducing the problem to sub-problems of lower order. There are two keys observations in this regard.

First, suppose there is a state variable, say $x_{j}$, such that $x_{j}$ does not appear in the system functions $f$ nor the cost function $f_{0}$, except for possibly $f_{j}$, and the final value of $x_{j}$ is unspecified. Then from equation (24) and the transversality conditions, the differential equation for the corresponding $\lambda_{j}$ and its boundary condition are

$$
\dot{\lambda}_{j}=-\frac{\partial f_{j}}{\partial x_{j}} \lambda_{j}, \quad \lambda_{j}\left(t_{f}\right)=0
$$

The only solution to this linear differential equation for a finite value of $\partial f_{j} / \partial x_{j}$ is $\lambda_{j} \equiv 0$. Thus, from equation (23), we see that the $j^{\text {th }}$ state equation does not influence the optimal control; this equation has uncoupled from the problem and may be integrated after the optimal control problem has been solved. This is the reason, for example, that the range equation uncouples from the other equations in the minimum time-to-climb problem.

Second, suppose that there is only one state equation ( $\underline{x}$ is a scalar) and one control variable:

$$
\dot{x}=f(x, u)
$$

with cost functional

$$
J=\int_{0}^{t_{f}} \phi(x, u) d t
$$

We have then, from equations (23) and (24)

$$
\begin{aligned}
& H=-\phi+\lambda f \\
& \dot{\lambda}=\frac{\partial \phi}{\partial x}-\lambda \frac{\partial f}{\partial x}
\end{aligned}
$$

The maximum principle gives, assuming that unbounded optimal control exists,

$$
\begin{aligned}
& H=-\phi+\lambda f=0 \\
& \frac{\partial H}{\partial u}=-\frac{\partial \phi}{\partial u}+\lambda \frac{\partial f}{\partial u}=0
\end{aligned}
$$


Eliminating $\lambda$ from these two equations gives

$$
-\frac{\partial \phi}{\partial u} f+\frac{\partial f}{\partial u} \phi=0
$$

This may be thought of as an equation for $u$ as a function of $x$, i.e., a feedback control law.

Alternatively, a direct approach may be used. Combining equations (25) and (26) gives

$$
J=\int_{0}^{t_{f}} \frac{\phi}{f} d x
$$

Thus $(\phi / f)$ is to be minimized with respect to $u$ holding $x$ fixed. Carrying out this minimization for unbounded control results in exactly equation (27). Actually, a stronger result holds for the single state case; if $\underline{u}$ is a bounded control of several components, then the optimal control is given by ${ }^{2,3}$

$$
\underline{u}=\underset{\underline{u} \in U}{\arg \min }\left(\frac{\phi}{f}\right)_{x=\text { const }}
$$

\section{Singular Perturbations and Time Scaling}

We have just seen that if the dynamic system can be approximated by a single state equation, or by a series of such equations, then the solution may be obtained by elementary means, without solving the 2PBVP. Singular perturbation theory provides a framework for accomplishing this, and indeed many of the references cited in the Introduction use this approach.

The extensive literature on the application of singular perturbation theory to optimal control problems in general and flight path optimization in particular will only be reviewed briefly here.

Perturbation methods have a long history of application in applied mathematics. Noteworthy examples are viscous fluid flow, nonlinear oscillations, and orbital dynamics. Singular perturbation methods were put on a solid mathematical foundation for ordinary differential equations by Tikonov ${ }^{29}$ and Vasileva ${ }^{30}$. Initial applications to control were by $\mathrm{O}^{\prime} \mathrm{Malley}^{31}$ and Kokotovic ${ }^{32}$. The theory concerns differential equations which depend on a parameter in such a way that the solutions as the parameter tends to zero do not approach uniformly the solution with the parameter set to zero.

The regions of nonuniform convergence are modeled by "boundary-layer" equations, a term arising in fluid dynamics. Solutions in the outer regions (away from the boundary layers) and the inner regions (the boundary layers) are independently determined by expanding all system variables in asymptotic power series. These solutions are then "matched" to determine their constants of integration. The final step is to combine the solutions to give uniformly valid approximations to the solution of the original problem. Thus the procedure is termed the method of matched asymptotic expansion (MAE).

Experience has shown that for the highly dynamic maneuvers of high performance fighter/attack type aircraft, carrying out the expansions to first order is required for high accuracy (see refs. 7 and 8 for 
example). For low performance aircraft, such as commercial transports, however, zero order analysis has been found to suffice (refs. 15-17 for example). The exception, for supersonic aircraft, is the rapid altitude transition typically occurring at transonic speeds; study of this transition is one of the main objectives of this report and will be taken up in detail later.

In this report, for the most part, we will consider only zero-order approximations and complete time-scale decoupling. For this simple case the elaborate procedures of the MAE method are trivial ${ }^{8}$ and do not need to be further explained.

Reference 33 was the first to suggest complete time-scale decoupling and to recognize its advantages. In this approach, a "small" parameter $\epsilon$ is inserted into the equations of motion as follows:

$$
\begin{gathered}
\dot{x}_{0}=f_{0}(\underline{x}, \underline{u}) \\
\epsilon \dot{x}_{1}=f_{1}(\underline{x}, \underline{u}) \\
\vdots \\
\epsilon^{n} \dot{x}_{n}=f_{n}(\underline{x}, \underline{u})
\end{gathered}
$$

or

$$
\epsilon^{i} \dot{x}_{i}=f_{i}(\underline{x}, \underline{u}) ; \quad i=0, \cdot, n
$$

where now $\underline{x}=\left(x_{0}, x_{1}, \cdot \cdot x_{n}\right)$. The maximum principle for the system (29) is the same as before, but with (see Theorem 5.1 of ref. 8)

$$
\begin{gathered}
H=\lambda_{0} \phi+\sum_{i=0}^{n} \lambda_{i} f_{i} \\
\epsilon^{i} \dot{\lambda}_{i}=-\frac{\partial H}{\partial x_{i}} ; \quad i=0, \cdot, n
\end{gathered}
$$

The $i^{\text {th }}$ dynamics are obtained by the stretching transformation $t_{i}=t / \epsilon^{i}$. Substituting and then setting $\epsilon=0$ gives (where now the dot denotes differentiation with respect to $t_{i}$ )

$$
\begin{aligned}
& \dot{x}_{0}=0 \quad \Longrightarrow x_{0}=\text { const. } \\
& \vdots \\
& \dot{x}_{i-1}=0 \quad \Longrightarrow x_{i-1}=\text { const. } \\
& \dot{x}_{i}=f_{i} \\
& 0=f_{i+1} \\
& \quad \vdots \\
& 0=f_{n}
\end{aligned}
$$


Thus the variables on a slower time-scale than $x_{i}$ are held constant and the variables on a faster timescale than $x_{i}$ have their system functions set to zero. In order to be able to apply the maximum principle to this single-state problem, the conditions of Theorem 5.3 of reference 8 must hold. Let $\underline{f}_{f}=\left(f_{i+1}, \cdots, f_{n}\right)$ and $\underline{x}_{f}=\left(x_{i+1}, \cdot \cdot, x_{n}\right)$. Then the key condition is that the matrix

$$
\left[\frac{\partial \underline{f}_{f}}{\partial \underline{x}_{f}}, \frac{\partial \underline{f}_{f}}{\partial \underline{u}}\right]
$$

have maximum rank evaluated along the solution.

If condition (33) is satisfied, then by the implicit function theorem the equations $\underline{0}=\underline{f}_{f}$ can be solved for $n-i$ of the components of $\underline{x}_{f}$ and $\underline{u}$ in terms of the remaining $m$. After substituting these solutions into $\dot{x}_{i}=f_{i}$, the optimal control may be determined directly from equation (28) with $f_{i}$ replacing $f$. Alternatively, the equations $\underline{0}=\underline{f}_{f}$ may be adjoined with ordinary Lagrange multipliers to the Hamiltonian function and the maximum principle applied. This latter method has the advantage that it provides the values of these multipliers. This is of interest because these multipliers are the slow estimates of the adjoint variables associated with the fast states ${ }^{8}$.

In the following section, transport aircraft guidance laws will be developed using the following time-scale dynamic model associated with equations (19):

$$
\begin{aligned}
& \dot{m}=-\epsilon \beta \\
& \dot{x}=v \\
& \epsilon \dot{E}=v F \\
& \epsilon^{2} \dot{f}=f_{h} v \gamma+f_{v} g(F-\gamma) \\
& \epsilon^{2} \dot{\gamma}=\frac{g}{v}(N-1)
\end{aligned}
$$

Note that with this formulation the mass is constant on all time-scales to zero order. The implications of this will be discussed later.

Note also that the system is not completely time-scale decoupled because $f$ and $\gamma$ are on the same time-scale. This was the approach adopted by Ardema (with $h$ replacing $f)^{7,8}$. Calise, on the other hand, time-scale decoupled $h$ and $\gamma^{2,3,4,33}$. This will be discussed in more detail later.

As a cost functional, following Erzberger a weighted sum of flight time and fuel consumption is adopted $^{15-17}$.

$$
J=\int_{0}^{t_{f}}\left(K_{1}+K_{2} \beta\right) d t
$$


Since some elements of transport airplane direct operating cost are time dependent and some are fuel consumption dependent, a proper weighting of these two effects by appropriate selection of the parameters $K_{1}$ and $K_{2}$ will give a close approximation of direct operating cost.

Finally, note that the system dynamics do not depend on state variable $x$ and that therefore the state equation $\dot{x}=v$ would uncouple from the problem if its terminal condition were not specified. 


\section{GUIDANCE LAW DEVELOPMENT}

\section{Range Dynamics}

Setting $\epsilon=0$ in equations (34) gives the range dynamics:

$$
\begin{aligned}
\dot{m} & =0 \\
\dot{x} & =v \\
0 & =v F \\
0 & =f_{h} v \gamma+f_{v} g(F-\gamma) \\
0 & =\frac{g}{v}(N-1)
\end{aligned}
$$

Thus the single state equation with its boundary conditions is

$$
\dot{x}=v, \quad x(0)=0, \quad x\left(t_{f}\right)=R
$$

subject to

$$
\begin{aligned}
& m=\text { const } \\
& F=0 \\
& \gamma=0 \\
& N=1
\end{aligned}
$$

The matrix (33) evaluated for conditions (38) is

$$
\left[\begin{array}{cccc}
\imath F_{E} & v F_{f} & 0 & v F_{\alpha} \\
f_{\imath} g F_{E} & f_{v} g F_{f} & f_{h} v-f_{v} g & f_{v} g F_{\alpha} \\
\frac{g}{v} N_{E} & \frac{g}{v} N_{f} & 0 & \frac{g}{v} N_{\alpha}
\end{array}\right]
$$

where, if $Q$ is any function of $E, f$, and $\alpha$,

$$
Q_{E}=\left.\frac{\partial Q}{\partial E}\right|_{f, \alpha}, \quad Q_{f}=\left.\frac{\partial Q}{\partial f}\right|_{E, \alpha}, \quad Q_{\alpha}=\left.\frac{\partial Q}{\partial \alpha}\right|_{f, E}
$$

The rank of matrix (39) depends on the energy dynamics solution, which determines $f$. For example, if the energy dynamics solution is on a terrain limit, then $f=h-h_{T}$ so that $f_{h}=1$ and $f_{v}=0$. Thus 
the matrix (39) becomes

$$
\left[\begin{array}{cccc}
v F_{E} & v F_{f} & 0 & v F_{\alpha} \\
0 & 0 & v & 0 \\
\frac{g}{v} N_{E} & \frac{g}{v} N_{f} & 0 & \frac{g}{v} N_{\alpha}
\end{array}\right]
$$

For the special case of thrust-aligned-with velocity, $N=L / W$ and $N$ may be taken as the control; the matrix now becomes, with $h$ replacing $f$,

$$
\left[\begin{array}{cccc}
v F_{E} & v F_{h} & 0 & v F_{N} \\
0 & 0 & v & 0 \\
0 & 0 & 0 & \frac{g}{v}
\end{array}\right]
$$

Clearly this will have maximum rank if either $F_{E} \neq 0$ or $F_{h} \neq 0$.

Assuming that matrix (39) has maximum rank, we may apply the maximum principle to the single state problem defined in equation (37). Although equation (28) could be used to directly determine the optimal control, because the adjoint $\lambda_{x}$ will be needed we proceed by forming the Hamiltonian. Note that the constraints (10) are now control constraints and do not need to be adjoined to the Hamiltonian.

Forming the Hamiltonian (see eqs. (23), (35), and (37)):

$$
H=-K_{1}-K_{2} \beta+\lambda_{x} v
$$

subject to $F=0, N=1$ and equations (10). Applying the maximum principle gives the optimal control as

$$
h_{c}, E_{c}=\underset{h, E}{\arg \min }\left(\frac{K_{1}+K_{2} \beta}{v}\right) \begin{aligned}
& F=0 \\
& N=1
\end{aligned}
$$

and the value of $\lambda_{x}$ as

$$
\lambda_{x}=\frac{K_{1}+K_{2} \beta_{c}}{v_{c}}
$$

Equation (42) defines the optimal cruise conditions.

There are two interesting special cases. First, if $K_{1}=1$ and $K_{2}=0$, the problem reduces to

$$
v_{c}=\max (v)
$$


as expected for minimum time. Second, if $K_{1}=0$ and $K_{2}=1$ and the fuel-flow varies linearly with throttle near the cruise point, equation (42) is equivalent to

$$
h_{c}, E_{c}=\arg \max _{h, E}\left[\frac{v(L / D)}{C}\right]
$$

where $C$ is the thrust specific fuel consumption. That is, the Brequet factor is to be maximized.

The total range of a transport aircraft is the sum of the ranges covered during the ascent, cruise, and descent portions of the flight. In our analysis of the range dynamics, the ascent and descent portions of the flight occur on a faster time scale and thus do not appear in the determination of the cruise condition.

In Erzberger's analysis of this problem ${ }^{15-17}$ he subtracts out the range covered in climb and descent in determining the cruise conditions. This is important in short range flight and in fact Erzberger was able to get good results for flight ranges short enough to be composed entirely of climb and descent. For the long range flights of supersonic transports, of primary interest here, this factor is of less importance. In the context of singular perturbation theory, climb and descent range may be expected to appear as first order corrections.

The range dynamics solution assumes constant mass. Variations in mass between take-off and cruise when determining the cruise point may be expected to be accounted for by first order corrections, not pursued here.

\section{Energy Dynamics}

Changing the independent variable to $t_{1}=t / \epsilon$ in equations (34) and then setting $\epsilon=0$ gives (the dot will denote differentiation with respect to $t_{1}$ in this section)

$$
\begin{aligned}
& m=\text { const } \\
& x=\text { const } \\
& \dot{E}=v F \\
& \gamma=\frac{F}{1-\frac{v}{g} \frac{f_{h}}{f_{v}}} \\
& N=1
\end{aligned}
$$

The matrix (33) for this case is

$$
\left[\begin{array}{ccc}
A & f_{h} v-f_{v} g & f_{v} g F_{\alpha} \\
\frac{g}{v} N_{f} & 0 & \frac{g}{v} N_{\alpha}
\end{array}\right]
$$


where

$$
\begin{aligned}
A=\frac{F}{f_{v} g-f_{h} v} & {\left[v f_{h h} h_{f} f_{v} g+v g f_{h v} v_{f} f_{v}+f_{h} v_{f} f_{v} g\right.} \\
& \left.\quad-f_{v h} h_{f} g f_{h} v-f_{v v} v_{f} g f_{h} v+\frac{f_{v} g\left(F_{h} h_{f}+F_{v} v_{f}\right)}{\bar{F}}\left(f_{v} g-f_{h} v\right)\right]
\end{aligned}
$$

For the case of solution on a terrain limit, $f=h-h_{T}, f_{h}=1, f_{v}=0, f_{h h}=f_{v v}=f_{h v}=0$ so that (47) becomes

$$
\left[\begin{array}{ccc}
0 & v & 0 \\
\frac{g}{v} N_{f} & 0 & \frac{g}{v} N_{\alpha}
\end{array}\right]
$$

For the special case of thrust-aligned with velocity vector and $N$ replacing $\alpha$ as control, this reduces to

$$
\left[\begin{array}{ccc}
0 & v & 0 \\
0 & 0 & \frac{g}{v}
\end{array}\right]
$$

which is in agreement with Section 6.2 of reference 8 , and clearly has maximum rank if $v \neq 0$.

Forming the Hamiltonian associated with equations (46):

$$
H=-K_{1}-K_{2} \beta+\lambda_{x} v+\lambda_{E} v F
$$

The constraints (10) are state-dependent control constraints for this problem. Maximizing $H$ gives

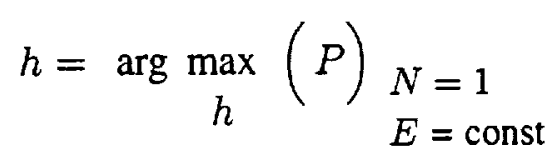

where

$$
P=\frac{v F}{K_{1}+K_{2} \beta-\lambda_{x} v}
$$

with the value of $\lambda_{E}$ as

$$
\lambda_{E}=\frac{1}{P}
$$

Note that as $h$ and $v$ approach $h_{c}$ and $v_{c}, P$ becomes infinitely large. The three terms in the denominator of $P$ have the following obvious interpretation. In climb, three factors are important: minimizing time $\left(K_{1}\right.$ term), minimizing fuel consumption $\left(K_{2}\right)$, and covering range $\left(-\lambda_{x} v\right)$. 
For the case of an unbounded local maximum, equation (49) implies

$$
\left(\frac{\partial P}{\partial h}\right)_{E}=0
$$

or, in terms of $v$ and $h$ (see eqs. (5)),

$$
P_{v}-\frac{v}{g} P_{h}=0
$$

For this case,

$$
\begin{gathered}
f(v, h)=P_{v}-\frac{v}{g} P_{h} \\
f_{h}=P_{v h}-\frac{v}{g} P_{h h} \\
f_{v}=P_{v v}-\frac{1}{g} P_{h}-\frac{v}{g} P_{h v}
\end{gathered}
$$

Substituting into the third of equations (46),

$$
\gamma=\frac{F}{1+\frac{v\left(g P_{v h}-v P_{h h}\right)}{g\left(v P_{v h}+P_{h}-g P_{v v}\right)}}
$$

As mentioned earlier, it is probably best to avoid computing numerical second derivatives and use equation (18):

$$
\gamma=\frac{F}{1+\frac{v}{g} \frac{d v}{d h}}
$$

instead for the value of $\gamma$ along the energy dynamics solution.

\section{Fast States Dynamics}

Changing the independent variable to $t_{2}=t / \epsilon^{2}$ in equations (34) and then setting $\epsilon=0$ gives (dot denotes differentiation with respect to $t_{2}$ ):

$$
\begin{aligned}
& m=\text { const. } \\
& x=\text { const. } \\
& E=\text { const. } \\
& \dot{f}=f_{h} v \gamma+f_{v} g(F-\gamma) \\
& \dot{\gamma}=\frac{g}{v}(N-1)
\end{aligned}
$$


Our main interest in this paper is to use these equations to model the altitude transition that typically occurs transonically in the energy dynamics solution for supersonic aircraft. There have been three approaches to the solution of equations (54).

Ardema ${ }^{7,8}$ for the case of $f=h$, left $h$ and $\gamma$ on the same time scale and iteratively solved the associated 2PBVP. Although this is not the approach that will be used here, the problem is formulated in general in Appendix $C$ as a starting point for future investigation. Calise ${ }^{33,34}$ time-scaled decoupled $h$ and $\gamma$ and obtained non-iterative solutions for each. This required adding a penalty term on $\gamma$ to the cost function and a "constrained matching" technique.

The approach used in this paper is a non-optimal one that assumes the fast state dynamics occur at constant load factor, $N$. This is motivated by reference 22 which showed that the transonic altitude transitions occurring in discontinuous energy dynamics solutions consisted of a push-over followed by a push-up (see fig. 2 which is reproduced from ref. 22). Reference 12 modeled this load factor history by two constant load factor segments and obtained good results. Using a non-optimal approach to the fast dynamics is partially justified by the fact that these altitude transitions take relatively little time and consume relatively little fuel.

One way to approximate the altitude transition is to begin flying a constant minimum load factor flight path when a jump is detected and then switch to a constant maximum load factor when the new branch is crossed; this is the dotted path in figure 3, from reference 12. This is undesirable for two reasons. First, the transition is initiated too late, and second, the transition path overshoots the new branch of the energy dynamics solution. In our approach, we use the fast state dynamics to determine $\bar{v}$, the optimum point for transition through $\bar{E}$ (see fig. 3).

Noting that $F=0$ because $\dot{E}=0$, consider the last two of equations (54)

$$
\begin{aligned}
& \dot{f}=f_{h} v \gamma-f_{v} g \gamma \\
& \dot{\gamma}=\frac{g K}{v}
\end{aligned}
$$

where $K=N-1$ is a known constant.

Following reference 12 , the first of these equations is divided by the second to give an equation in $f$ and $\gamma$ :

$$
\begin{gathered}
\frac{d f}{d \gamma}=\frac{v}{g K}\left(f_{h} v-f_{v} g\right) \gamma \\
g K \int \frac{d f}{v\left(v f_{h}-g f_{v}\right)}=\int \gamma d \gamma
\end{gathered}
$$

From equation (9) for $E=$ const., $d h=-\frac{v}{g} d v$ so that

$$
d f=f_{h} d h+f_{v} d v=\left(f_{v}-f_{h} \frac{v}{g}\right) d v
$$


Substituting into equation (56) and carrying out the integration gives

$$
\begin{gathered}
-K \int \frac{d v}{v}=\int \gamma d \gamma+\text { const. } \\
-K \ln v=\frac{1}{2} \gamma^{2}+\text { const. }
\end{gathered}
$$

Now label the last point on the subsonic climb path as point 1, the first point on the supersonic climb path as point 2 , and the load factor transition point by an overbar (see fig. 3 ). Then equation (57) must hold from point 1 to the transition point with $K_{1}=N_{1}-1$ and from the transition to point 2 with $K_{2}=N_{2}-1$ :

$$
\begin{aligned}
& -K_{1} \ln \frac{\bar{v}_{A}}{v_{1}}=\frac{1}{2}\left(\bar{\gamma}^{2}-\gamma_{1}^{2}\right) \\
& -K_{2} \ln \frac{v_{2}}{\bar{v}_{A}}=\frac{1}{2}\left(\gamma_{2}^{2}-\bar{\gamma}^{2}\right)
\end{aligned}
$$

Solving for $\bar{v}$ and $\bar{\gamma}$ :

$$
\begin{gathered}
\bar{v}_{A}=\left[\frac{v_{2}^{K_{2}}}{v_{1}^{K_{1}}} e^{\left.\left(\frac{\gamma_{2}^{2}-\gamma_{1}^{2}}{2}\right)\right]^{\frac{1}{K_{2}-K_{1}}}}\right. \\
\bar{\gamma}_{A}=\sqrt{\frac{2\left(K_{1} \gamma_{2}^{2}-K_{2} \gamma_{1}^{2}\right)+4 K_{1} K_{2} \ln \frac{v_{2}}{v_{1}}}{2\left(K_{1}-K_{2}\right)}}
\end{gathered}
$$

This is the same solution as obtained in reference 12 except that now the values of $\gamma_{1}$ and $\gamma_{2}$ are to be determined according to equation (15).

The transition path is then determined as follows. Constant load factor solutions are generated with load factor $N_{1}$ (see Appendix B) which leave the lower energy branch of the climb path at different points. The solution that just achieves $v=\bar{v}$ when $E=\bar{E}$ is chosen and then the load factor is set to $N_{2}$ for the transition from $\bar{E}$ to the higher energy branch.

It is also possible to obtain an integrated solution if the small $\gamma$ assumption is not made (this was not possible in reference 12 because of Coriolis and Earth curvature terms). This may be of importance because $\gamma$ may become large in some altitude transitions. Now divide the last two of equations (17) 
and integrate with $F=0$ :

$$
\begin{aligned}
& \frac{d f}{d \gamma}=\frac{v\left(f_{h} v-f_{v} g\right) \sin \gamma}{g(N-\cos \gamma)} \\
& -\int \frac{d v}{v}=\int \frac{\sin \gamma}{N-\cos \gamma} d \gamma+\text { const. } \\
& -\ln v=\ln (N-\cos \gamma)+\text { const. } \\
& v(N-\cos \gamma)=\text { const. }
\end{aligned}
$$

Applying this to both branches of the transition

$$
\begin{aligned}
& \bar{v}_{B}\left(N_{1}-\cos \bar{\gamma}\right)=v_{1}\left(N_{1}-\cos \gamma_{1}\right) \\
& \bar{v}_{B}\left(N_{2}-\cos \bar{\gamma}\right)=v_{2}\left(N_{2}-\cos \gamma_{2}\right)
\end{aligned}
$$

or, solving for $\bar{v}$ and $\bar{\gamma}$,

$$
\begin{gathered}
\bar{\tau}_{B}=\frac{v_{2}\left(N_{2}-\cos \gamma_{2}\right)-v_{1}\left(N_{1}-\cos \gamma_{1}\right)}{N_{2}-N_{1}} \\
\bar{\gamma}_{B}=\cos ^{-1}\left[\frac{v_{2} N_{1}\left(N_{2}-\cos \gamma_{2}\right)-v_{1} N_{2}\left(N_{1}-\cos \gamma_{1}\right)}{v_{2}\left(N_{2}-\cos \gamma_{2}\right)-v_{1}\left(N_{1}-\cos \gamma_{1}\right)}\right]
\end{gathered}
$$

Comparison of equations (58) and (59) shows that the non-small $\gamma$ solution, $\bar{v}_{B}$, is just as easy to implement as the small $;$ solution, $\bar{v}_{A}$. Also note that, as a check,

$$
\begin{aligned}
& \lim _{N_{1} \rightarrow \infty} \bar{v}_{A}=\lim _{N_{1} \rightarrow \infty} \bar{v}_{B}=v_{1} \\
& \lim _{N_{2} \rightarrow \infty} \bar{v}_{A}=\lim _{N_{2} \rightarrow \infty} \bar{v}_{B}=v_{2}
\end{aligned}
$$




\section{NUMERICAL EXAMPLE}

The guidance algorithm developed in the previous section has been implemented in the ACSYNT Computer Code and used to compute near-optimal trajectories for a supersonic transport design. The main characteristics of the design are listed in table 1.

Figure 4 shows maximum thrust of the aircraft as a function of Mach number for various energy levels (recall that a linear throttle is not assumed), and figure 5 shows the total drag for $N=1$ as a function of Mach for various energy levels, in the region of Mach 1. The transonic drag rise is clearly shown in figure 5 , and this raises the possibility that there may be an instantaneous altitude transition in the energy climb path near Mach 1.

The first step of the algorithm is to find the optimum cruise point, as given by equation (42). Figure 6 shows the optimal cruise point at each energy level throughout the flight envelope for minimum fuel ( $K_{1}=0$ and $K_{2}=1$ ). The optimal cruise point is interior to the flight envelope except from about Mach 1.25 to Mach 1.75, for which it is on the loft ceiling bound.

Figure 7 shows the data of figure 6 plotted in a different way, as $\lambda_{x}$ vs. Mach (see eq. (43)). This curve has three local minimums, each a locally optimal cruise point. One of these is a subsonic condition at Mach 0.95. The globally optimum point is at Mach 2.4, the highest Mach allowed. From figure 6, this Mach 2.4 cruise point is at an altitude of about 52,500 ft. The Mach 2.4 cruise condition has about a $15 \%$ higher cruise efficiency than the Mach 0.95 condition, as measured by $\lambda_{x}$; the Mach 0.95 cruise point would be used for over-land flight.

The next step in the algorithm is determining the climb path. This involves maximizing $P$ (see eqs. (49) and (50)) with respect to $h$ at energy levels from take-off to cruise. Figure 8 plots $P$ as a function of Mach for various energy levels for maximum thrust and again minimum fuel. The maximum dynamic pressure constraint is not applied for this calculation. The value of $\lambda_{x}$ used in equation (50) is given by equation (43) for the Mach 2.4 optimal cruise condition. It is seen that for many energy levels $P$ has two or more local maxima in the vicinity of Mach 1; it is the jumping of the global maxima between these local maxima that causes the transonic altitude transition.

The resulting flight path in the Mach-altitude plane is shown in figure 9. The path starts along a terrain limit and then climbs at almost a constant high subsonic Mach. At about 32,500 ft, it instantaneously transitions to about $22,500 \mathrm{ft}$ at Mach 1.25 . It then continues up to the cruise point, with a jump to higher altitudes between Mach 1.6 and 1.8. Also shown in figure 9 is the path with the dynamic pressure constraint imposed. It is seen that the unconstrained path violates the constraint by only a small amount between Mach 1.3 and 1.7 .

Figure 10 compares the minimum fuel flight path with $\lambda_{x}$ included in $P$ in equation (50) with the path with the $\lambda_{x}$ term omitted. The latter case corresponds to minimum fuel to climb without regard to a range constraint. The paths are similar except at high speed where the path with $\lambda_{x}$ omitted has much higher dynamic pressure (there is no dynamic pressure constraint imposed). A computation was made to verify that including the $\lambda_{x}$ term gives better performance. Referring to figure 11 , the path with the $\lambda_{x}$ term included ended with an airplane weight of $657,310 \mathrm{lb}$ and a range of $836 \mathrm{~nm}$. The path without $\lambda_{x}$ ended at $690,683 \mathrm{lb}$ and $349 \mathrm{~nm}$. By the Brequet formula, the range covered in a cruise condition 
is (see eq. (45)):

$$
R_{\text {cruise }}=\frac{v(L / D)}{C} \ln \frac{m_{0}}{m_{f}}
$$

At the cruise condition, $v=2323 \mathrm{ft} / \mathrm{sec},(L / D)=9.0$, and $C=1.315 \mathrm{Ib}_{\text {fuel }}$ per hour per $\mathrm{lb}_{\text {thrust }}$. Thus for the same fuel consumed along the path with $\lambda_{x}$, the path without $\lambda_{x}$, has a range of

$$
349+\frac{(9.0)(2323)}{(1.315)}\left(\frac{3600}{6076}\right) \ln \frac{690,683}{657,310}=815 \mathrm{~nm}
$$

Thus the case with $\lambda_{x}$ gives $21 \mathrm{~nm}$. more range for the same fuel than the case without $\lambda_{x}$.

Minimum fuel, minimum time $\left(K_{1}=0, K_{2}=1\right)$, and "minimum direct operating cost" climb trajectories are compared in figure 12 . For the minimum cost trajectory, $K_{1}=\$ 500 / \mathrm{hr}$ and $K_{2}=$ $\$ 0.0626 / \mathrm{lb}$; these are the values used in reference 15 for short range subsonic transports, and would likely need to be adjusted for supersonic long range transports. The minimum fuel and minimum time trajectories are quite different. The latter has no transonic altitude transition, whereas the former has a large one. Also, the minimum time path is much lower in altitude in the high supersonic range (the dynamic pressure constraint was relaxed for this calculation and would be violated by the minimum time path). As expected, the minimum cost path is intermediate between the other two, being more like the minimum time path.

One of the principal goals of this research has been to develop an algorithm for computing the trajectory segments connecting the branches of the energy climb path in the transonic region, that is, the altitude transitions. Specifically, equation (50) was used to determine $\bar{v}$, the value of $v$ which is to be obtained when $E=\bar{E}$ (see fig. 3). An iteration is then made to determine where on the subsonic branch of the ECP the departure should be made to achieve this condition. The constant load factor integration as described in Appendix B is used to generate the flight paths.

Figure 13 shows the transition for $N_{1}=0.97$ and $N_{2}=1.05$ for the minimum fuel case in the altitude-Mach plane, and figure 14 shows the same path in the transonic region. The integration is terminated when the flight path angle is equal to the flight path angle on the supersonic branch of the ECP as given by equation (53). The dynamic pressure limit was ignored for this calculation. The figures show that there is a very close match between the altitude transition and the ECP at the termination of the former, and that even mild maneuvers $\left(N_{1}\right.$ and $N_{2}$ close to 1) give adequate transition trajectories.

The transition trajectories for the same conditions, but using the linear estimate of $\bar{v}$ as given by equation (58), are shown in figure 15. Comparing figures 14 and 15 shows that the nonlinear solution gives a better match with the supersonic branch of the ECP than does the linear.

The transition trajectory for a more severe load factor maneuver, $N_{1}=0.5$ and $N_{2}=1.5$, is shown in figure 16 (these load factors would not be acceptable for a commercial transport). As compared with a more benign maneuver, as shown in figure 14 , the transition through $\bar{E}$ occurs at a much higher altitude and the trajectory is much closer to $\bar{E}$, as expected.

Figure 17 shows the variation of energy rate, $v F$, as a function of Mach in the transonic region for the mild transition $\left(N_{1}=0.97, N_{2}=1.05\right)$. As expected, the energy rate drops when the load factor is 
switched from 0.97 to 1.05 , but never gets near zero. Also as expected, the flight path angle, $\gamma$, at first decreases, and then increases when the load factor is switched as shown in figure 18; the magnitude of $\gamma$ stays below $6 \mathrm{deg}$, making the small $\gamma$ approximation extremely good.

The same plots are made for the more severe maneuver $\left(N_{1}=0.5, N_{2}=1.5\right)$ in figures 19 and 20. In this case, the energy rate becomes negative after the load factor switch and the magnitude of $\gamma$ reaches about $22 \mathrm{deg}$, meaning that equations (59) and not equations (58) should be used for the calculation of the transition point. 


\section{CONCLUDING REMARKS}

An algorithm for optimizing supersonic transport trajectories suitable for use in an aircraft synthesis computer code has been developed. The algorithm has been implemented in the ACSYNT computer program and illustrated using a typical supersonic transport design.

The algorithm is based on singular perturbation theory and complete time-scale decoupling of the energy-state version of the equations of motion (except for the fast dynamics). This results in replacing the functional optimization problem by a series of function optimization problems.

The first problem is determining the optimal cruise condition. This involves a weighted sum of the importance of time and fuel consumption. The second problem is determining the energy-climb path (ECP) to the cruise condition, which involves a weighted sum of the importance of time, fuel consumption, and cruise efficiency.

For the fast dynamics, a variable is introduced such that the ECP gives a consistent value of the flight path angle. This variable is left on the same time scale as the flight path angle and a nonoptimal solution of the fast dynamics using constant load factor segments is obtained.

Numerical results for a nominal supersonic transport showed the following: (1) The optimal cruise point was at the highest and fastest point in the flight envelope, although there are local optimal cruise points at high subsonic and low supersonic speeds. (2) The ECP for the minimum fuel case had a large transonic altitude transition, the minimum time case had no transition, and the minimum direct operating cost case had a mild transition. (3) The altitude transition solutions gave good matches between the subsonic and supersonic branches of the ECP with operationally acceptable load factors.

There are two obvious shortcomings of the present state of the analysis. First, the weight is held constant during the search for the optimal cruise point. This weight is the gross take-off weight according to the time-scale assumptions, but in practice could be some empirical estimate of the weight at the start of cruise. Second, the range during climb and descent is ignored when optimizing the cruise point. This is obviously a more serious problem at short ranges than for the long ranges of a supersonic transport.

It is expected that both of these shortcomings could be eliminated by solving the time-scaled equations of motion to first order, that is, by expanding all the state variables to first order terms. This is the next obvious step in this research. In addition to solving these problems, the first order solutions will give better overall accuracy to the algorithm. 


\section{APPENDIX A - NOMENCLATURE}

$D=$ drag

$E=$ mechanical energy

$F=$ normalized tangential force

$g=$ gravity

$h=$ altitude

$H=$ Hamiltonian

$J=$ cost functional

$L=$ lift

$m=$ mass

$M=$ Mach number

$q=$ dynamic pressure

$T=$ thrust

$v=$ velocity

$x=$ range

$\alpha=$ angle of attack

$\beta=$ fuel flow rate

$\epsilon=$ small parameter

$\gamma=$ flight path angle

$\lambda=$ adjoint variable

$\phi=$ cost function

$\rho=$ air density 


\section{APPENDIX B - NUMERICAL INTEGRATION OF STATE EQUATIONS}

In this appendix we give the algorithms by which the state equations are integrated within ACSYNT. Two cases are of interest. First, the integration of the trajectory when pairs of altitude and energy (equivalently altitude and speed or Mach number) are given; $\left(E_{0}, h_{0}\right),\left(E_{1}, h_{1}\right), \cdots,\left(E_{f}, h_{f}\right)$. This is sometimes called path following. Second, the integration of the trajectory when the normal load factor $N$ is held constant.

\section{Path Following}

It is assumed that all variables are known at step $n-1$. These values are sought at step $n$, knowing only $E_{n}$ and $h_{n}$. Of particular interest are the values of $t_{n}, m_{n}$, and $x_{n}$. We start with equations (7), with $\dot{\gamma}=0$, written in finite difference form from step $n-1$ to step $n$ :

$$
\begin{gathered}
\frac{\Delta m}{\Delta t}=-\bar{\beta} \\
\frac{\Delta x}{\Delta t}=\bar{v} \cos \bar{\gamma} \\
\frac{\Delta E}{\Delta t}=\frac{\bar{v} \bar{F}^{\prime}}{g \bar{m}} \\
\frac{\Delta h}{\Delta t}=\bar{v} \sin \bar{\gamma} \\
0=\frac{g}{\bar{v}}(\bar{N}-\cos \bar{\gamma})
\end{gathered}
$$

where $F^{\prime}=T \cos \alpha-D$ and, if $Q$ is any variable,

$$
\begin{aligned}
& \Delta Q=Q_{n}-Q_{n-1} \\
& \bar{Q}=\frac{Q_{n}+Q_{n-1}}{2}
\end{aligned}
$$

From the first of equations (B1),

$$
m_{n}=m_{n-1}-\bar{\beta} \Delta t
$$

so that

$$
\bar{m}=m_{n-1}-\frac{\bar{\beta}}{2} \Delta t
$$

Substituting this into the third of equations (B1) and solving for $\Delta t$,

$$
\Delta t=\frac{m_{n-1}}{\frac{\bar{v} \bar{F}^{\prime}}{g \Delta E}+\frac{\bar{\beta}}{2}}
$$

Note that $\bar{v}, \Delta E, \bar{h}$, and $\bar{\beta}$ are all known (the latter if throttle is fixed). The only quantity not known in equation (B3) is $\bar{F}^{\prime}$, which depends on $\alpha$. If $\Delta t$ were known, the fourth of equations (B1) gives $\bar{\gamma}$ :

$$
\bar{\gamma}=\sin ^{-1}\left(\frac{\Delta h}{\bar{v} \Delta t}\right)
$$


The algorithm may now be stated as follows:

1. Guess $\alpha_{n}$.

2. Compute $\Delta t$ from equation (B3).

3. Compute $\bar{\gamma}$ from equation (B4).

4. Check to see if the fifth of equations (B1) is satisfied to a suitable degree of accuracy. If not, select a new $\alpha_{n}$ by a suitable one-dimensional search procedure and return to step (2). If satisfied, continue.

5. Compute $m_{n}$ from equation (B2) and $t_{n}$ and $x_{n}$ from:

$$
\begin{aligned}
& t_{n}=t_{n-1}+\Delta t \\
& x_{n}=x_{n-1}+\bar{v} \Delta t \cos \bar{\gamma}
\end{aligned}
$$

Phillips ${ }^{35}$ has proposed an alternative integration scheme as follows. The third of equations (B1) is now averaged directly

$$
\frac{\Delta E}{\Delta t}=\frac{1}{2 g}\left(\frac{v_{n} F_{n}^{\prime}}{m_{n}}+\frac{v_{n-1} F_{n-1}^{\prime}}{m_{n-1}}\right)
$$

This is then combined with equation (B2) to give

$$
\left(\frac{\bar{\beta} F_{n-1} v_{n-1}}{g m_{n-1}}\right) \Delta t^{2}-\left(F_{n} v_{n}+F_{n-1} v_{n-1}+2 \bar{\beta} \Delta E\right) \Delta t+2 g m_{n-1} \Delta E=0
$$

This is a quadratic equation to be solved for $\Delta t$, and replaces equation (B3) in the numerical procedure. As the integration step size tends to zero, these two integration schemes become equivalent.

\section{Constant Normal Load Factor $(N)$ Paths}

In this case, $\Delta E$ is not a suitable integration variable because it may happen that $\Delta E \leq 0$, which causes serious numerical problems. Alternative choices are $\Delta t$ and $\Delta \gamma$. Because the choice $\Delta t$ results in an algorithm with three nested iterations, we follow Phillips ${ }^{35}$ and choose $\Delta \gamma$. For this integration we do not neglect $\dot{\gamma}$.

Because $N, \Delta \gamma$, and $\gamma_{n}$ are now known,

$$
\bar{K}=g(N-\cos \bar{\gamma})
$$

is a known constant. Thus the finite difference form of the last of equations (8) is

$$
\frac{\Delta \gamma}{\Delta t}=\frac{\bar{K}}{\bar{v}}
$$


Use this and equation (B2) to eliminate $m_{n}$ and $\Delta t$ from the rest of equations (B1). The result is

$$
\begin{gathered}
h_{n}=h_{n-1}+\left(\frac{\Delta \gamma \sin \bar{\gamma}}{\bar{K}}\right)\left(\frac{v_{n}+v_{n-1}}{2}\right)^{2} \\
\Delta E=\frac{\bar{v}^{2} \bar{F}^{\prime 2}}{\bar{K}\left(\frac{g m_{n-1}}{\Delta \gamma}-\frac{\bar{\beta} \bar{v}}{2 \bar{K}}\right)} \\
0=N\left(g m_{n-1}-\frac{\bar{\beta} \bar{v} \Delta \gamma}{\bar{K}}\right)-\bar{T} \sin \bar{\alpha}-\bar{L}
\end{gathered}
$$

The algorithm is as follows:

(1) Guess $v_{n}$.

(2) Solve for $h_{n}$ from equation (B5).

(3) Compute $E_{n}=h_{n}+\frac{1}{2 g} v_{n}^{2}$ and $\Delta E=E_{n}-E_{n-1}$.

(4) Guess $\alpha_{n}$.

(5) Check to see if equation (B7) is satisfied. If not, select a new $\alpha_{n}$ and repeat this step. If satisfied, continue.

(6) Check to see if equation (B6) is satisfied. If not, select a new $v_{n}$ and return to step (2). If satisfied, continue.

(7) Compute all other quantities of interest.

Thus this algorithm requires a nested two parameter search, whereas the path following routine required a one parameter search. From equations (B5)-(B7) it is seen that $\bar{K}=0(N=\cos \bar{\gamma})$ is not allowed. Should this happen, one solution is a $\Delta t$ integration but, as mentioned earlier, this involves a three parameter search.

Phillips ${ }^{35}$ has proposed an alternative method of constant load factor integration with $\Delta \gamma$ as integration variable. This approach holds all variables constant at the previous step but does a second order integration of the altitude state equation. The increments $\Delta t$ and $\Delta v$ may be now directly computed from the third and fifth of equations (3):

$$
\begin{gathered}
\Delta t=\frac{v_{n-1}}{g\left(N-\cos \gamma_{n-1}\right)} \Delta \gamma \\
\Delta v=g\left(F_{n-1}-\sin \gamma_{n-1}\right) \Delta t
\end{gathered}
$$

Differentiating the fourth of equations (3):

$$
\ddot{h}=\dot{V} \sin \gamma+v \dot{\gamma} \cos \gamma
$$


Using the third and fifth of equations (3) this becomes

$$
\ddot{h}=g\left(F_{n-1} \sin \gamma_{n-1}+N \cos \gamma_{n-1}-1\right)=C
$$

Integrating twice:

$$
h=\frac{1}{2} C t^{2}+c_{1} t
$$

The constant of integration $c_{1}$ is determined from $\dot{h}_{n-1}=v_{n-1} \sin \gamma_{n-1}=c_{1}$ so that

$$
h_{n}=v_{n-1} \Delta t \sin \gamma_{n-1}+\frac{1}{2} g \Delta t^{2}\left(F_{n-1} \sin \gamma_{n-1}+N \cos \gamma_{n-1}-1\right)
$$

with $\Delta t$ determined as above. Reference 35 shows that this gives good numerical results. 


\section{APPENDIX C - NECESSARY CONDITIONS FOR FAST DYNAMICS}

The state equations of the fast dynamics are the last two of equations (54):

$$
\begin{aligned}
& \dot{f}=f_{h} v \gamma+f_{v} g(F-\gamma) \\
& \dot{\gamma}=\frac{g}{v}(N-1)
\end{aligned}
$$

with $m, x$, and $E$ (the slower states) all known constants; the control variable is $\alpha$.

From equation (23) the Hamiltonian is

$$
\begin{aligned}
H=- & K_{1}-K_{2} \beta+\lambda_{x} v+\lambda_{E} v F+\lambda_{f}\left[f_{h} v \gamma+f_{v} g(F-\gamma)\right] \\
& +\lambda_{\gamma} \frac{g}{v}(N-1)+\sum_{i=1}^{5} \nu_{i} s_{i}
\end{aligned}
$$

where the constraints equations (11) are now state constraints and must be adjoined to $H$ with multipliers $\nu_{i}$; the $s_{i}$ are assumed to be written as functions of $f$ and $E$, the latter a known constant. The adjoints $\lambda_{x}$ and $\lambda_{E}$ are known constants from the slower dynamics solutions, equations (43) and (51). From equation (24) the adjoint equations are

$$
\begin{gathered}
\dot{\lambda}_{f}=K_{2} \beta_{f}-\lambda_{x} v_{f}-\lambda_{E} v_{f} F-\lambda_{E} v F_{f}-\lambda_{f}\left[\left(f_{h}\right)_{f} v \gamma+f_{h} v_{f} \gamma+f_{v} g F_{f}\right. \\
\left.\quad+\left(f_{v}\right)_{f} g(F-\gamma)\right]+\lambda_{\gamma} \frac{g}{v^{2}} v_{f}(N-1)-\lambda_{\gamma} \frac{g}{v} N_{f}-\sum_{i=1}^{5} \nu_{i} s_{f_{i}} \\
\dot{\lambda}_{\gamma}=-\lambda_{f} f_{h} v+\lambda_{f} f_{v} g
\end{gathered}
$$

where the notations of equations (5) and (40) have been used. In these equations, if $Q(h, v)$ is any function then

$$
Q_{f}=Q_{h} h_{f}+Q_{v} v_{f}
$$

Assuming an unbounded optimal control, conditions (a) and (b) of the maximum principle give

$$
\begin{aligned}
& \lambda_{E} v F_{\alpha}+\lambda_{f} f_{v} g F_{\alpha}+\lambda_{\gamma} \frac{g}{v} N_{\alpha}=0 \\
& -K_{1}-K_{2} \beta+\lambda_{x} v+\lambda_{E} v F+\lambda_{f}\left[f_{h} v \gamma+f_{v} g(F-\gamma)\right]+\lambda_{\gamma} \frac{g}{v}(N-1)=0
\end{aligned}
$$

From equations (2) explicit forms for $F_{\alpha}$ and $N_{\alpha}$ are

$$
\begin{aligned}
& F_{\alpha}=\frac{1}{m g}\left(T_{\alpha} \cos \alpha-T \sin \alpha-D_{\alpha}\right) \\
& N_{\alpha}=\frac{1}{m g}\left(T_{\alpha} \sin \alpha+T \cos \alpha+L_{\alpha}\right)
\end{aligned}
$$


Equations (C1), (C3), and (C5) are used to model transitions from an initial condition to the energy dynamics solution (energy climb path, or ECP), from the ECP to a terminal condition, and between different branches of the ECP if the ECP is discontinuous. In what follows, the first case, transition from an initial condition to the ECP will be considered for the purpose of illustration.

For this case, the boundary conditions on equations (C1) and (C3) are

$$
\begin{aligned}
& f(0)=f_{0} \\
& \gamma(0)=\gamma_{0} \\
& \lambda_{f}(0)=\frac{K_{1}+K_{2} \beta_{0}-\lambda_{x} v_{0}-\lambda_{E} v_{0} F_{0}-\lambda_{\gamma_{0}} \frac{g}{v_{0}}\left(N_{0}-1\right)}{f_{h_{0}} v_{0} \gamma_{0}+f_{v_{0}} g\left(F_{0}-\gamma_{0}\right)} \\
& \lambda_{\gamma}(0)=\lambda_{\gamma_{0}} \text { selected to match with ECP }
\end{aligned}
$$

where the second of equations (5) was used and where all quantities are known except $\lambda_{\gamma_{0}}$. In summary, equations (C1) and (C3) are to be integrated with control given by the first of equations (C5) subject to initial conditions equations (C7).

The fast dynamics equations depend on the nature of the ECP solution because this solution determines the choice of variable $f$. If the ECP solution is an unbounded optimum, singular perturbation theory states that the ECP solution will be an equilibrium point of the fast dynamics, ${ }^{7,8}$ and the goal is to find a solution of the fast dynamics such that the solution approaches the ECP as $t \rightarrow \infty$. If, on the other hand, the ECP is on a constraint, then the fast dynamics solution may reach the ECP in finite time. ${ }^{36}$

Some examples will nou be given. If the ECP is on a terrain limit,

$$
f=f(h, v)=h-h_{m}
$$

In this case the transformation $\left(h \cdot \imath^{\prime}\right) \rightarrow(E, f)$ and its inverse are given by

$$
\begin{array}{ll}
E=h+\frac{1}{2 g} v^{2} & h=f \\
f=h-h_{m} & v=\sqrt{2 g(E-f)}
\end{array}
$$

so that

$$
f_{h}=1, \quad f_{v}=0, \quad h_{f}=1, \quad v_{f}=-\frac{g}{v}
$$

and from equation (C4)

$$
Q_{f}=Q_{h}-\frac{g}{v} Q_{v}
$$


Putting these results into equations $(\mathrm{C} 1)$ and $(\mathrm{C} 3)$

$$
\begin{gathered}
\dot{f}=v \gamma \\
\dot{\gamma}=\frac{g}{v}(N-1) \\
\dot{\lambda}_{f}=K_{2}\left(\beta_{h}-\frac{g}{v} \beta_{v}\right)+\lambda_{x} \frac{g}{v}+\lambda_{E} \frac{g}{v} F-\lambda_{E} v\left(F_{h}-\frac{g}{v} F_{v}\right)+\lambda_{f} \frac{g}{v} \gamma \\
-\lambda_{\gamma} \frac{g^{2}}{v^{3}}(N-1)-\lambda_{\gamma} \frac{g}{v}\left(N_{h}-\frac{g}{v} N_{v}\right)-\sum_{i=1}^{5} \nu_{i}\left(s_{h}-\frac{g}{v} s_{v}\right)_{i} \\
\dot{\lambda}_{\gamma}=-\lambda_{f} v
\end{gathered}
$$

and into equations (C5)

$$
\begin{aligned}
& \lambda_{E} v F_{\alpha}+\lambda_{\gamma} \frac{g}{v} N_{\alpha}=0 \\
& -K_{1}-K_{2} \beta+\lambda_{x} v+\lambda_{E} v F+\lambda_{f} v \gamma+\lambda_{\gamma} \frac{g}{v}(N-1)=0
\end{aligned}
$$

The initial conditions for the integration of equations (C7) are as follows:

$$
\begin{aligned}
& f(0)=f_{0} \\
& \gamma(0)=\gamma_{0} \\
& \lambda_{f}(0)=\frac{K_{1}+K_{2} \beta_{0}-\lambda_{x} v_{0}-\lambda_{E} v_{0} F_{0}-\lambda_{\gamma_{0}} \frac{g}{v}\left(N_{0}-1\right)}{v_{0} \gamma_{0}} \\
& \lambda_{\gamma}(0)=\lambda_{\gamma_{0}}, \quad \text { selected to match with ECP }
\end{aligned}
$$

If the ECP is on a dynamic pressure limit, the transformation $(h, v) \rightarrow(E, f)$ is

$$
\begin{aligned}
& E=h+\frac{1}{2 g} v^{2} \\
& f=\frac{1}{2} \rho v^{2}-q_{m}
\end{aligned}
$$

with $\rho=\rho(h)$ so that

$$
f_{h}=\frac{1}{2} \rho_{h} v^{2}, \quad f_{v}=\rho v
$$

The inverse transformation is implicit. Taking differentials and using the fact that $E=$ const.:

$$
d E=d h+\frac{v}{g} d v=0
$$




$$
d f=\frac{1}{2} \rho_{h} v^{2} d h+\rho v d v
$$

Combining these equations gives

$$
\begin{gathered}
v_{f}=\frac{1}{v\left(\rho-\frac{\rho_{h} v^{2}}{2 g}\right)} \\
h_{f}=\frac{1}{\frac{1}{2} \rho_{h} v^{2}-\rho g}
\end{gathered}
$$

Then from equation (C4)

$$
Q_{f}=\frac{Q_{h}}{\frac{1}{2} \rho_{h} v^{2}-\rho g}+\frac{Q_{v}}{v\left(\rho-\frac{\rho_{h} v^{2}}{2 g}\right)}
$$

This gives, for example,

$$
\begin{gathered}
\rho_{f}=\frac{\rho_{h}}{\frac{1}{2} \rho_{h} v^{2}-\rho g} \\
\left(\rho_{h}\right)_{f}=\frac{\rho_{h h}}{\frac{1}{2} \rho_{h} v^{2}-\rho g} \\
\left(f_{h}\right)_{f}=\frac{\frac{1}{2} \rho_{h h} v^{2}}{\frac{1}{2} \rho_{h} v^{2}-\rho g}+\frac{\rho_{h} v}{v\left(\rho-\frac{p_{h} v^{2}}{2 g}\right)} \\
\left(f_{v}\right)_{f}=\frac{\rho_{h} v}{\frac{1}{2} \rho_{h} v^{2}-\rho g}+\frac{\rho}{v\left(\rho-\frac{\rho_{h} v^{2}}{2 g}\right)} \\
F_{f}=\frac{F_{h}}{\frac{1}{2} \rho_{h} v^{2}-\rho g}+\frac{F_{v}}{v\left(\rho-\frac{\rho_{h} v^{2}}{2 g}\right)} \\
\beta_{f}=\frac{\beta_{h}}{\frac{1}{2} \rho_{h} v^{2}-\rho g}+\frac{\beta_{v}}{v\left(\rho-\frac{\rho_{h} v^{2}}{2 g}\right)}
\end{gathered}
$$


Equations (C1) and (C3) become

$$
\begin{gathered}
\dot{f}=\frac{1}{2} \rho_{h} v^{3} \gamma+\rho v g(F-\gamma) \\
\dot{\gamma}=\frac{g}{v}(N-1) \\
\dot{\lambda}_{f}=K_{2} \beta_{f}-\lambda_{x} v_{f}-\lambda_{E} v_{f} F-\lambda_{E} v F_{f}-\lambda_{f}\left[\left(f_{h}\right)_{f} v \gamma+\frac{1}{2} \rho_{h} v^{2} v_{f} \gamma+\rho v g F_{f}\right. \\
\left.+\left(f_{v}\right)_{f} g(F-\gamma)\right]+\lambda_{\gamma} \frac{g}{v^{2}} v_{f}(N-1)-\lambda_{\gamma} \frac{g}{v} N_{f}-\sum_{i=1}^{5} \nu_{i} s_{f_{i}} \\
\dot{\lambda}_{\gamma}=-\lambda_{f} \frac{1}{2} \rho_{h} v^{3}+\lambda_{f} \rho v g
\end{gathered}
$$

and equations (C5) become

$$
\begin{gathered}
\lambda_{E} v F_{\alpha}+\lambda_{v} \rho v g F_{\alpha}+\lambda_{\gamma} \frac{g}{v} N_{\alpha}=0 \\
-K_{1}-K_{2} \beta+\lambda_{x} v^{v}+\lambda_{E} v F+\lambda_{f}\left[\frac{1}{2} \rho_{h} v^{3} \gamma+\rho v g(F-\gamma)\right]+\lambda_{\gamma} \frac{g}{v}(N-1)=0
\end{gathered}
$$

Initial conditions equations (C7) become

$$
\begin{aligned}
& f(0)=f_{0} \\
& \because(0)=\gamma_{0} \\
& \lambda_{f}(0)=\frac{K_{1}+K_{2} \beta_{0}-\lambda_{x} v_{0}-\lambda_{E} v_{0} F_{0}-\lambda_{\gamma_{0}} \frac{g}{v_{0}}\left(N_{0}-1\right)}{\frac{1}{2} \rho_{h_{0}} v_{0}^{3} \gamma_{0}+\rho_{0} v_{0} g\left(F_{0}-\gamma_{0}\right)} \\
& \lambda_{\gamma}(0)=\lambda_{\gamma_{0}} \text { selected to match with ECP }
\end{aligned}
$$

In all of these equations, quantities such as $v_{f}, F_{f}$, and $\left(f_{h}\right)_{f}$ are to be determined from the equations derived above.

Finally, consider the case for which the ECP is an unbounded local optimum. From equation (52), $f$ in this case is

$$
f=P_{v}-\frac{v}{g} P_{h}
$$


where $P$ is given by equation (50). Thus

$$
\begin{aligned}
& f_{h}=P_{v h}-\frac{v}{g} P_{h h} \\
& f_{v}=P_{v v}-\frac{1}{g} P_{h}-\frac{v}{g} P_{h v}
\end{aligned}
$$

Because $E=$ const.,

$$
\begin{aligned}
& d E=d h+\frac{v}{g} d v=0 \\
& d f=f_{h} d h+f_{v} d v
\end{aligned}
$$

so that

$$
\begin{aligned}
& h_{f}=\frac{1}{f_{h}-\frac{g f_{v}}{v}} \\
& v_{f}=\frac{1}{f_{v}-\frac{v f_{h}}{g}}
\end{aligned}
$$

Let $\phi=\phi(h, v)$ be defined as

$$
\phi=f_{v}-\frac{v f_{h}}{g}
$$

Then

$$
\phi=P_{v v}-\frac{1}{g} P_{h}-\frac{2 v}{g} P_{h v}+\frac{v^{2}}{g^{2}} P_{h h}
$$

and equation (C4) becomes

$$
Q_{f}=\frac{Q_{v}-\frac{v}{g} Q_{h}}{\phi}
$$

This explains how to compute quantities such as $\beta_{f}, F_{f}$, and $\left(f_{h}\right)_{f}$ in equations (C3). Equations (C1), (C3), (C5), and (C7) will not be written out explicitly for this case. 


\section{REFERENCES}

1. Myklebust, Arvid; and Gelhausen, P.: Putting the ACSYNT on Aircraft Design. Aerospace America, Sept. 1994, pp. 26-30.

2. Calise, A.: Singular Perturbation Methods for Variational Problems in Aircraft Flight. IEEE Transactions on Automatic Control, vol. AC-21, June 1976, pp. 345-353.

3. Calise, A.: Extended Energy Management Methods for Flight Performance Optimization. AIAA Journal, vol. 15, no. 3, Mar. 1977, pp. 314-321.

4. Calise, A.: A New Boundary Layer Matching Procedure for Singularly Perturbed Systems. IEEE Transaction on Automatic Control, vol. AC-23, no. 3, June 1978.

5. Kelley, H.: Aircraft Maneuver Optimization by Reduced-Order Approximation. Control and Dynamic Systems, vol. 10, Academic Press, New York, 1973, pp. 131-178.

6. Kelley, H.; and Edelbaum, T.: Energy Climbs, Energy Turns, and Asymptotic Expansions. Journal of Aircraft, vol. 7, Jan. 1970, pp. 93-95.

7. Ardema, M.: Solution of the Minimum Time-to-Climb Problem by Matched Asymptotic Expansions. AIAA Journal, vol. 14, no. 7, July 1976.

8. Ardema, M.: Singular Perturbations in Flight Mechanics. NASA TM X-62,380, Aug. 1974.

9. Shinar, J.: On Applications of Singular Perturbation Techniques in Nonlinear Optimal Control. Automatica, vol. 19, no. 2, 1983, pp. 203-211.

10. Bryson, A.; Desai, M.; and Hoffman, W.: Energy-State Approximation in Performance Optimization of Supersonic Aircraft. Journal of Aircraft, vol. 6, no. 6, Nov.-Dec. 1969.

11. Kelley, H.; Cliff, E.; and Weston, A.: Energy State Revisited. Optimal Control Applications \& Methods, vol. 7, 1986, pp. 195-200.

12. Ardema, M.; Bowles, J.; Terjesen, E.; and Whittaker T.: Approximate Altitude Transitions for High-Speed Aircraft. Journal of Guidance, Control, and Dynamics, vol. 18, no. 3, May-June 1995, pp. 561-566.

13. Ardema, M.; and Rajan, N.: Selection of Slow and Fast Variables in Three-Dimensional Flight Dynamics. American Control Conference, WP7-3:30, June 1984.

14. Ardema, M.; and Rajan, N.: Slow and Fast State Variables for Three-Dimensional Flight Dynamics. Journal of Guidance, Control, and Dynamics, vol. 8, no. 4, July-Aug. 1985.

15. Erzberger, H.: Automation of On-Board Flight path Management. NASA TM 84212, Dec. 1981.

16. Erzberger, H.: Theory and Applications of Optimal Control in Aerospace Systems. AGARDograph No. 251, July 1981.

17. Erzberger, H.; and Lee, H.: Algorithm for Fixed-Range Optimal Trajectories. NASA TP 1565, July 1980 . 
18. Breakwell, J.: Optimal Flight-Path-Angle Transitions in Minimum-Time Airplane Climbs. Journal of Aircraft, vol. 14, no. 8, Aug. 1977.

19. Breakwell, J.: More about Flight-Path-Angle Transitions in Optimal Airplane Climbs. Journal of Guidance and Control, vol. 1, no. 3, May-June 1978.

20. Weston, A.; Cliff, E.; and Kelley, H.: Altitude Transitions in Energy Climbs. Automatica, vol. 19, Mar. 1983, pp. 199-202.

21. Shinar, J.; and Fainstein, V.: Improved Feedback Algorithms for Optimal Maneuvers in Vertical Plane. AIAA Paper 85-1976, 1985.

22. Ardema, M.; and Yang, L.: Interior Transition Layers in Flight-Path Optimization. Journal of Guidance, vol. 11, no. 1, Jan.-Feb. 1988.

23. Ardema, M.; and Rajan, N.: Separation of Time Scales in Aircraft Trajectory Optimization. Journal of Guidance, Control, and Dynamics, vol. 8, no. 2, Mar.-Apr. 1985.

24. Bharadwaj, S.; Wu, M.; and Mease, K.: Identifying Time-Scale Structure for Simplified Guidance Law Development. AIAA Guidance, Navigation, and Control Conference, AIAA Paper 97-3708, 1997.

25. Bryson, A.; and Ho, Y.: Applied Optimal Control. Hemisphere Publishing Co., 1975.

26. Jacobson, E.; Lele, M.; and Speyer, J.: New Necessary Conditions of Optimality for Control Problems with State-Variable Inequality Constraints. Journal of Mathematical Analysis and Applications, vol. 35, Aug. 1971, pp. 255-284.

27. Pontryagin, L.; Boltyanskii, U.; Gamkrelidze, R.; and Mishchenko, E.: The Mathematical Theory of Optimal Processes. Interscience, 1962.

28. Leitmann, G.: The Calculus of Variations and Optimal Control. Plenum Press, New York and London, 1981.

29. Tihonov, A.: Systems of Differential Equations Containing Small Parameters Multiplying Some of the Derivatives. Math. Sb., vol. 73, no. 3, N.S. (31), 1952 (in Russian).

30. Vasileva, A.: Asymptotic Behavior of Solutions to Certain Problems Involving Nonlinear Differential Equations Containing a Small Parameter Multiplying the Highest Derivatives. Russian Math Surveys, vol. 18, no. 3, 1963.

31. O'Malley, R.: Introduction to Singular Perturbations: Academic Press, New York and London, 1974.

32. Kokotovic, P.; and Sannuti, P.: Singular Perturbation Method for Reducing the Model Order in Optimal Control Design. IEEE Trans. on Automatic Control, vol. 13, no. 4, Aug. 1968.

33. Calise, A.; Aggarwal, R.; and Goldstein, F.: Singular Perturbation Analysis of Optimal Flight Profiles for Transport Aircraft. Joint Automatic Control Conference, June 1977.

34. Calise, A.: Optimization of Aircraft Altitude and Flight-Path Angle Dynamics. Journal of Guidance, Control and Dynamics, vol. 7, no. 1, Jan.-Feb. 1984. 
35. Phillips, J.: An Accurate and Flexible Trajectory Analysis. Paper 975599, presented at the 1997 World Aviation Congress, Anaheim, CA, Oct. 1997.

36. Calise, A.; and Corban, J.: Optimal Control of Two-Time-Scale Systems with State-Variable Inequality Constraints. Journal of Guidance, Control, and Dynamics, vol. 15, no. 2, Mar.-Apr. 1992. 
Table 1. Characteristics of supersonic transport

\begin{tabular}{|l|c|}
\hline \hline Gross take-off weight & $753,500 \mathrm{lb}$ \\
Wing planform area & $5500 \mathrm{ft}^{2}$ \\
Wing span & $137.35 \mathrm{ft}$ \\
Leading edge sweep & $48 \mathrm{deg}$ \\
Aspect ratio & 3.43 \\
Body length & $314 \mathrm{ft}$ \\
Payload & \\
first class passengers & 30 \\
coach class passengers & 274 \\
flight crew & 2 \\
flight attendants & 9 \\
Maximum Mach number & 2.4 \\
Maximum dynamic pressure & $1000 \mathrm{psf}$ \\
\hline
\end{tabular}

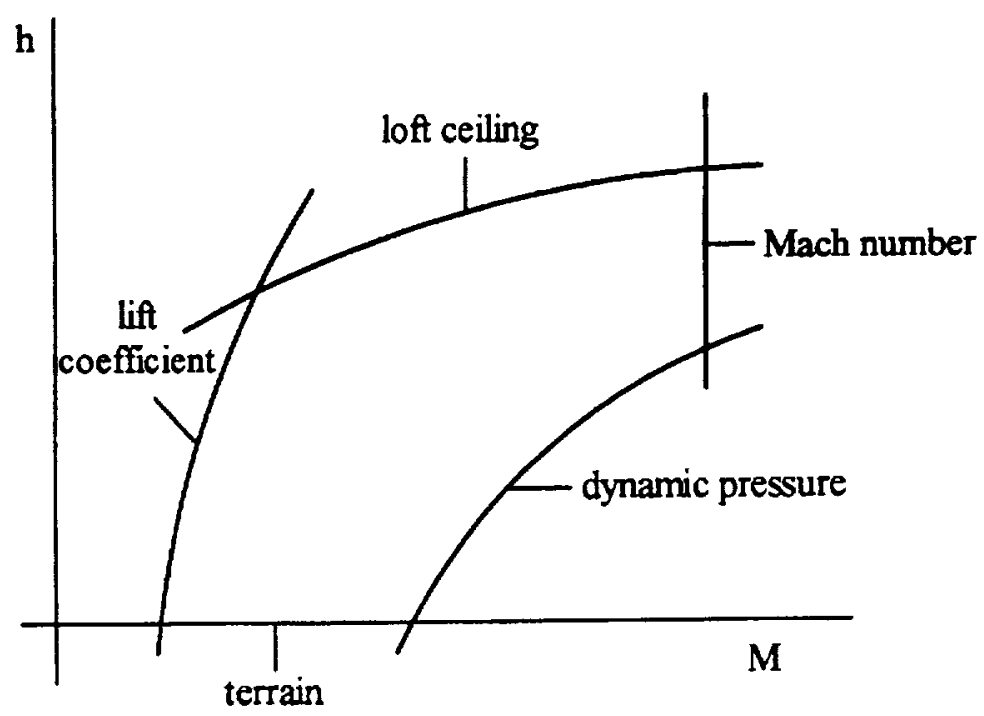

Figure 1. Sketch of constraints defining flight envelope. 


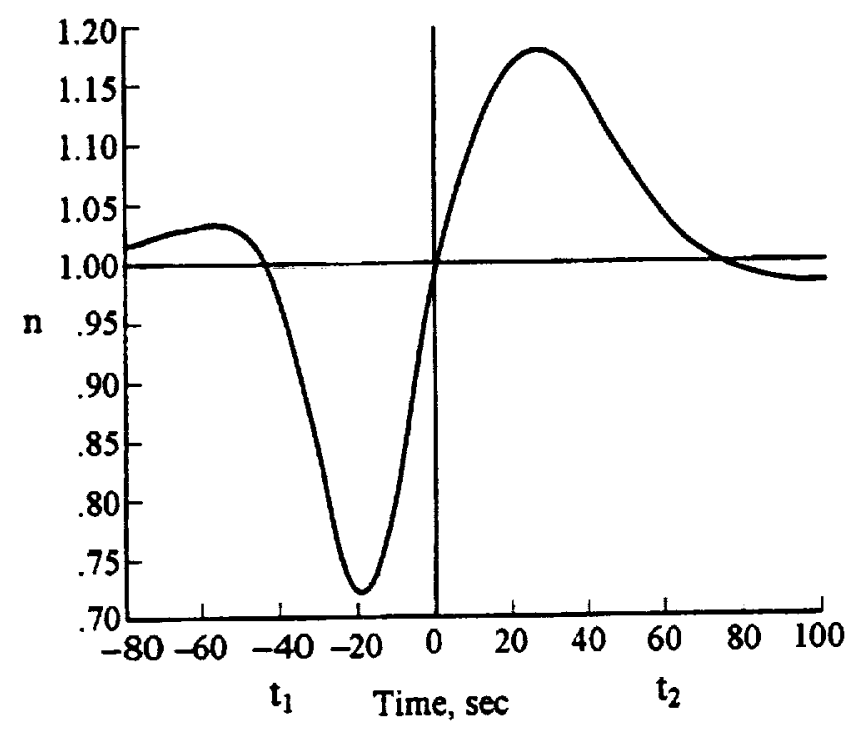

Figure 2. Load factor history during altitude transition for a high performance aircraft.

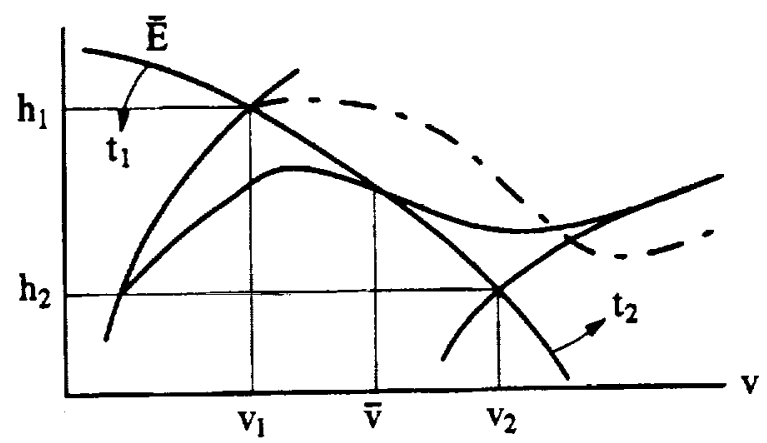

Figure 3. Sketch of an altitude transition. 


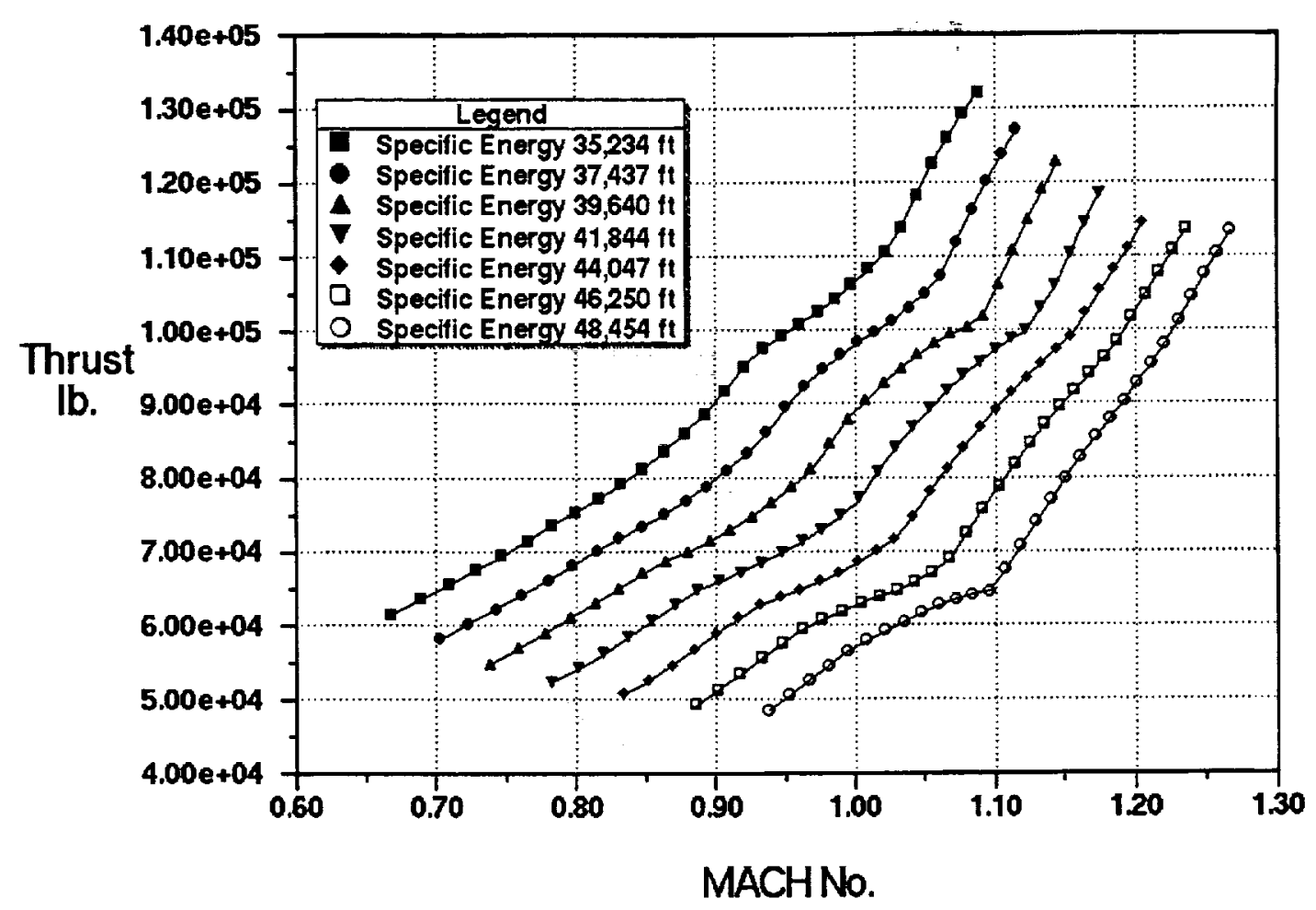

Figure 4. Thrust vs. Mach number in the transonic region.

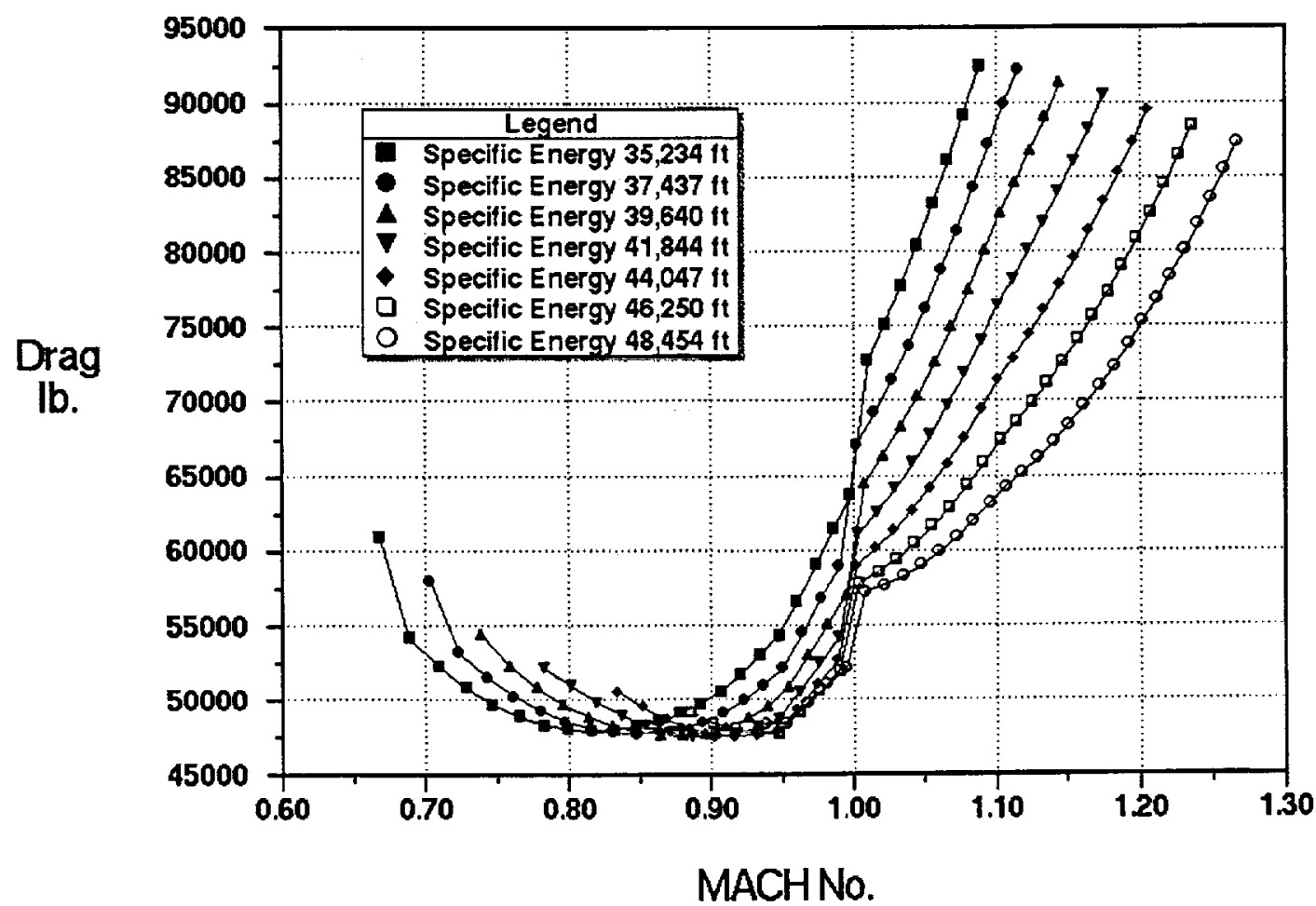

Figure 5. Drag for $N=1$ vs. Mach number in the transonic region. 


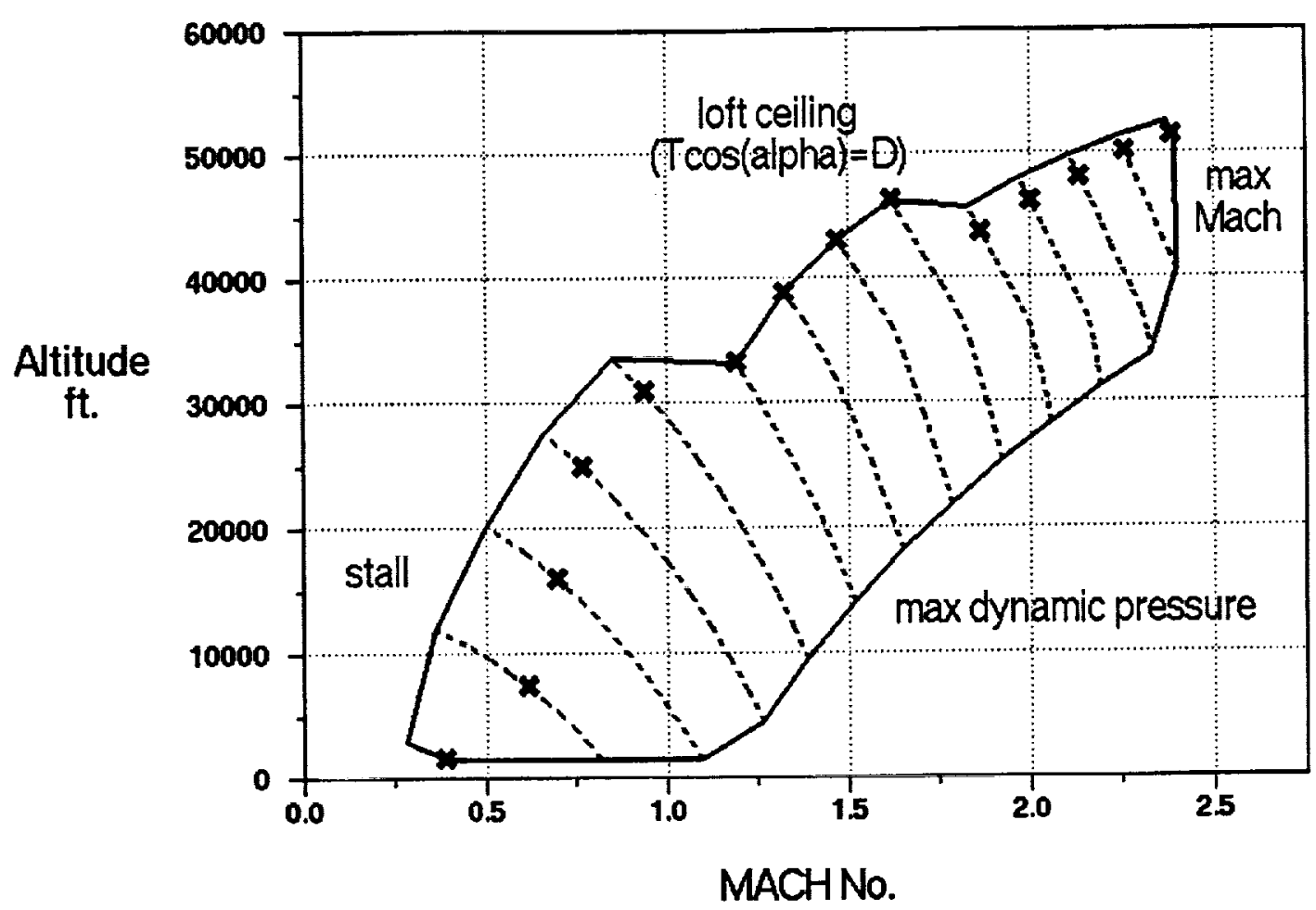

Figure 6. Optimum cruise points in the flight envelope for minimum fuel.

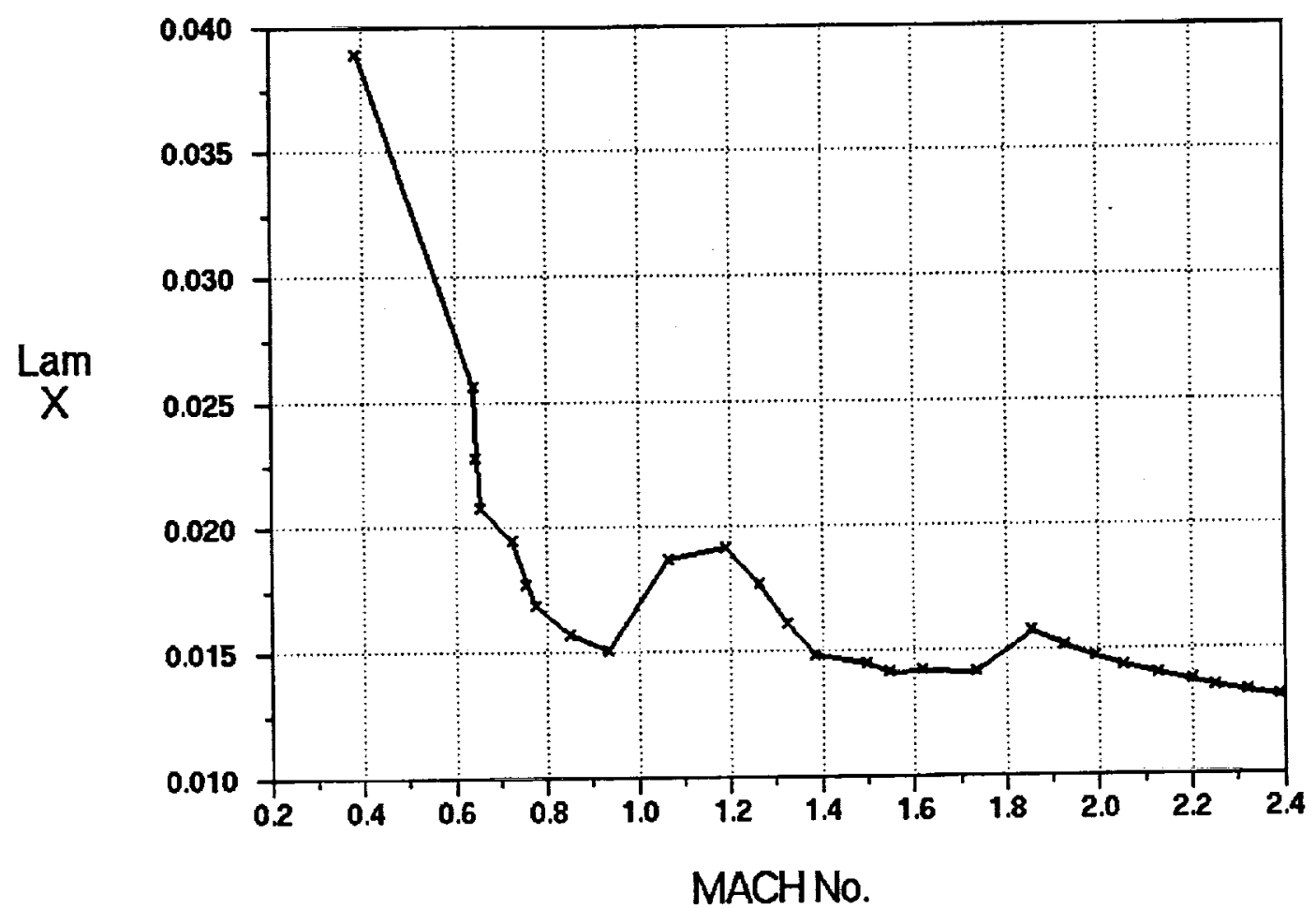

Figure 7. $\lambda_{x}$ vs. Mach number. 


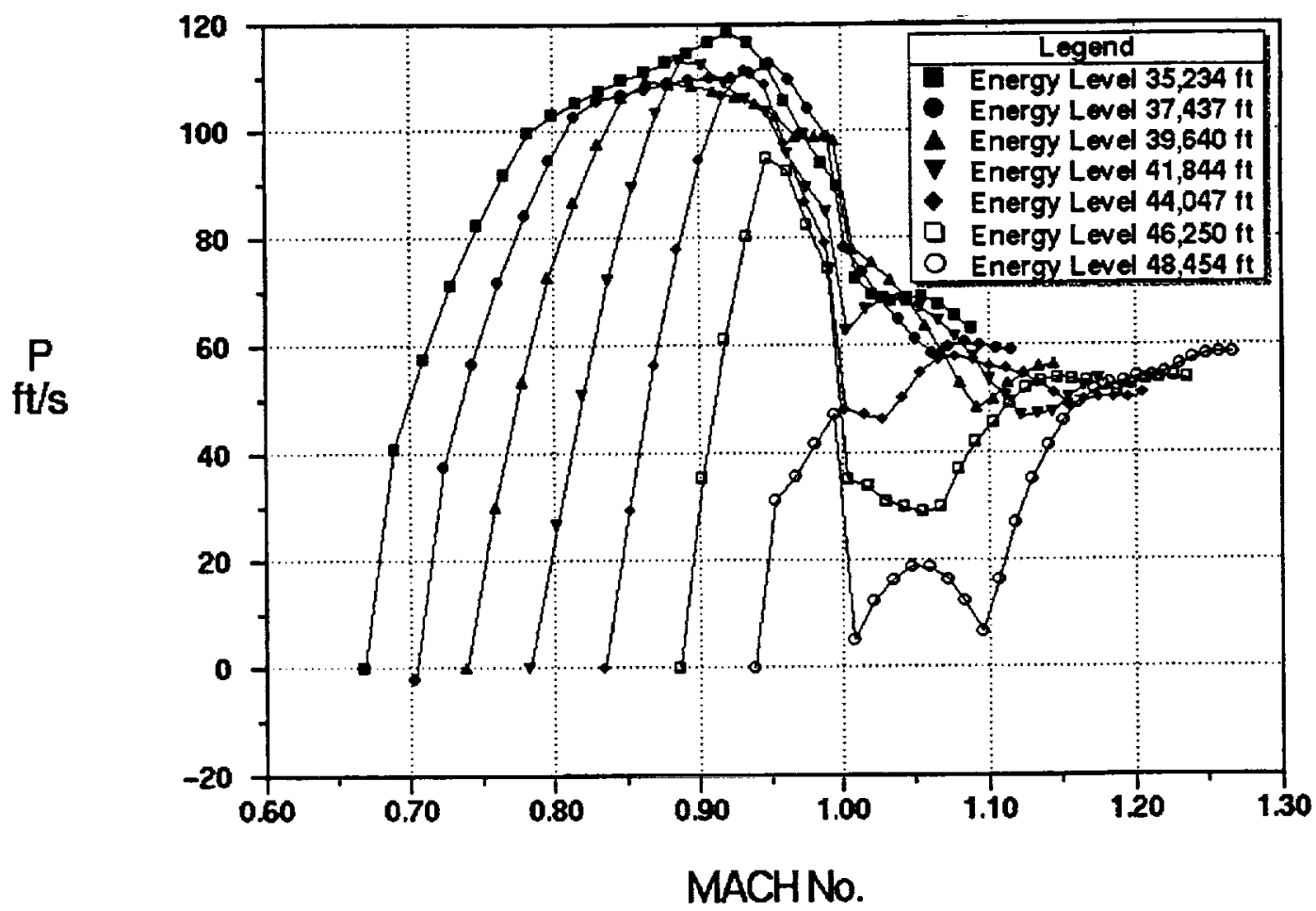

Figure 8. Energy rate vs. Mach number in the transonic region.

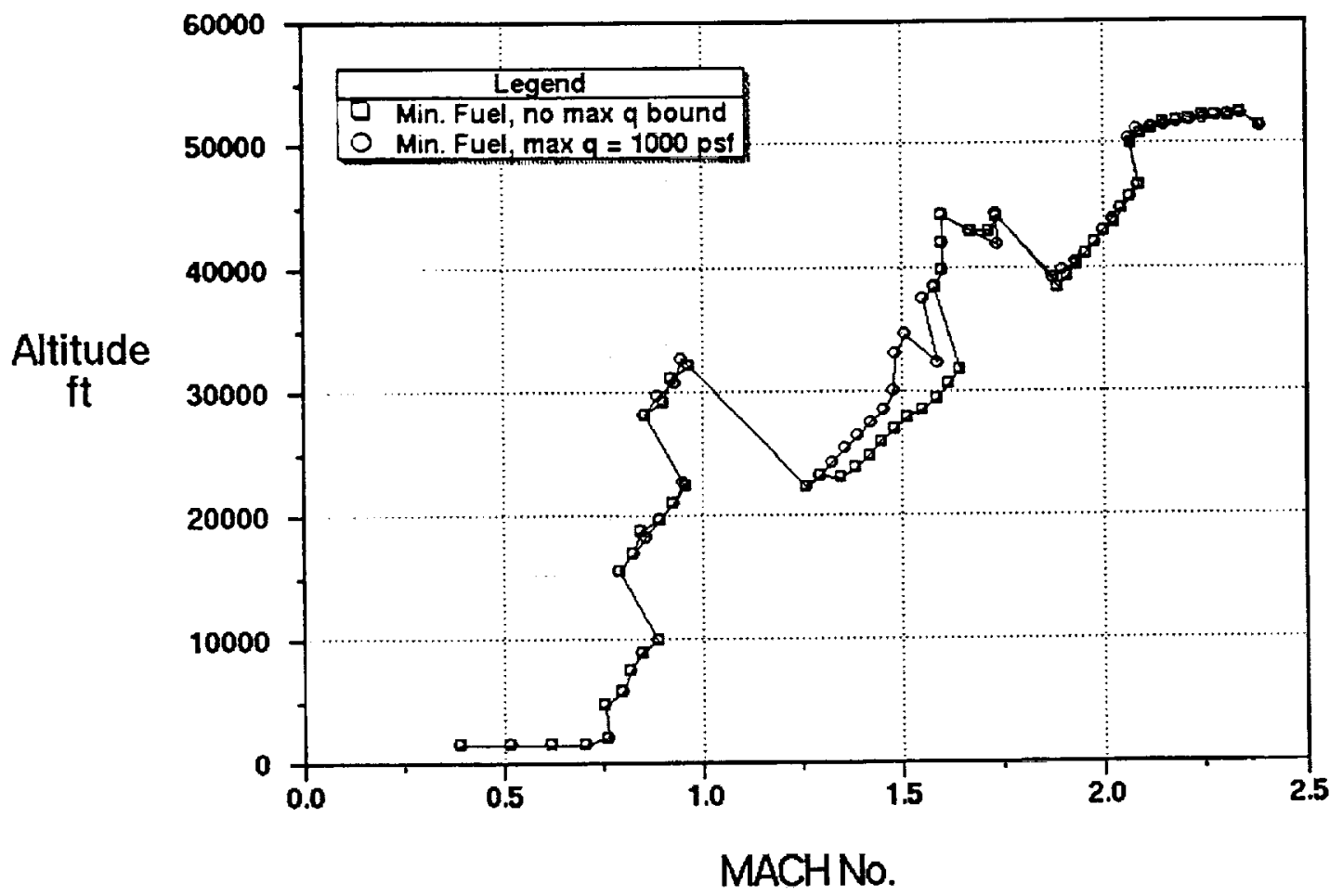

Figure 9. Energy climb path with and without dynamic pressure constraint. 


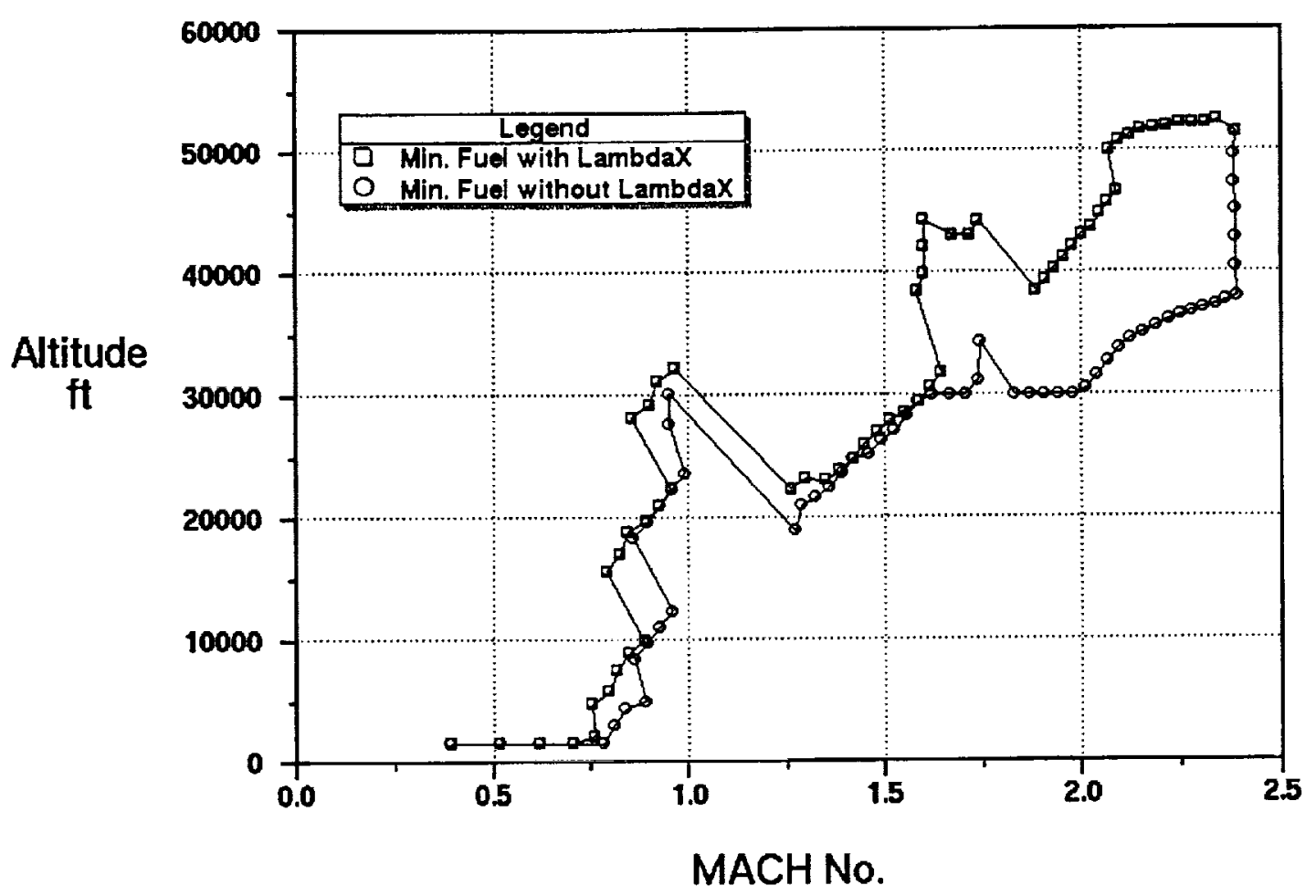

Figure 10. Energy climb path with and without $\lambda_{x}$.

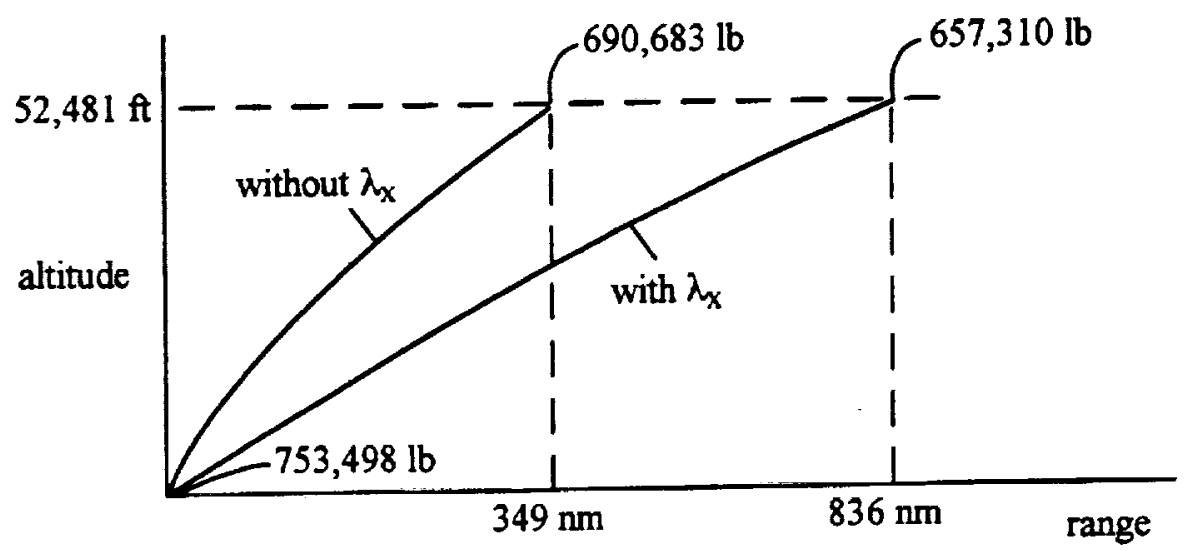

Figure 11. Sketch of trajectories with and without $\lambda_{x}$. 


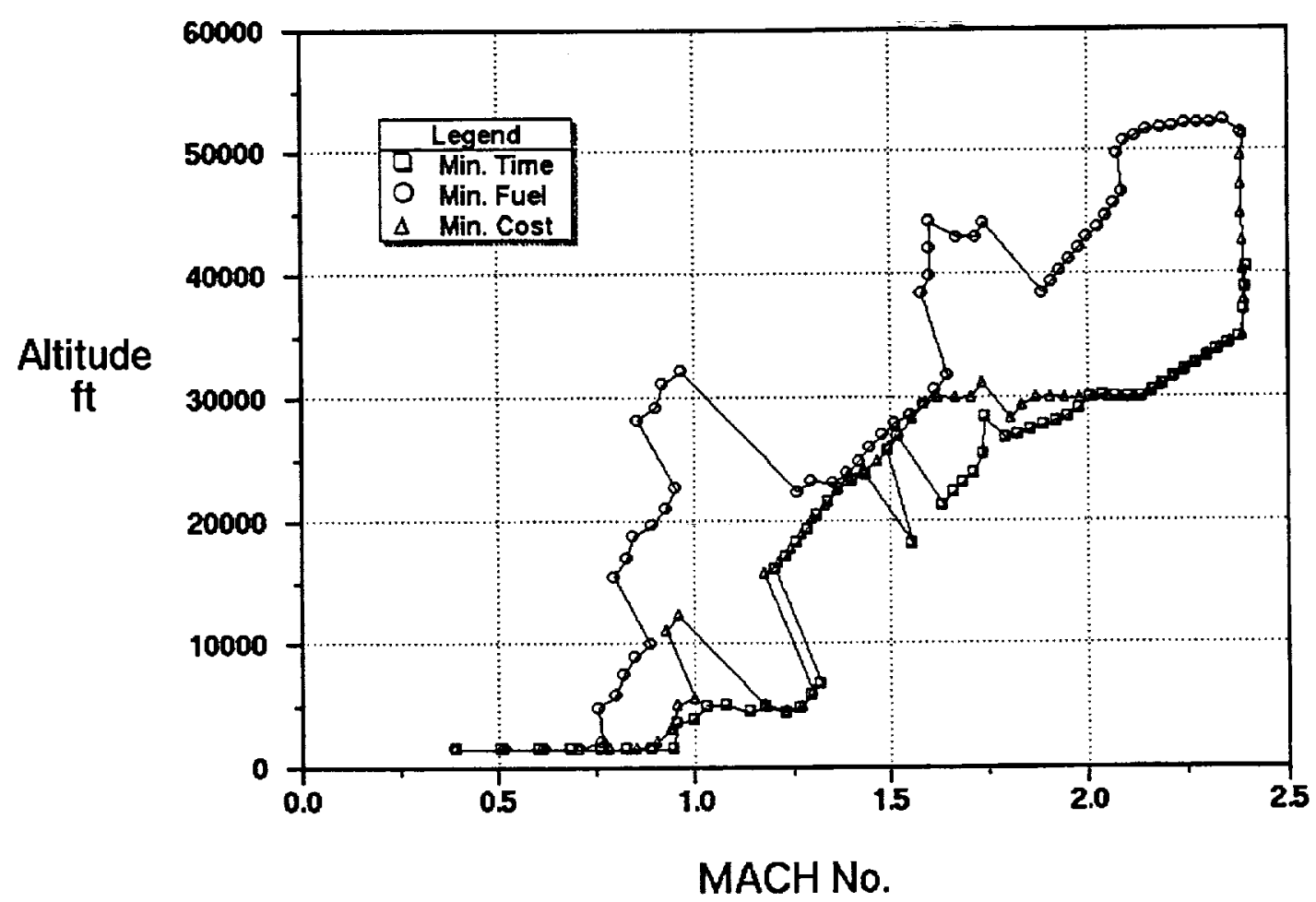

Figure 12. Energy climb path for minimum fuel, minimum time, and minimum cost.

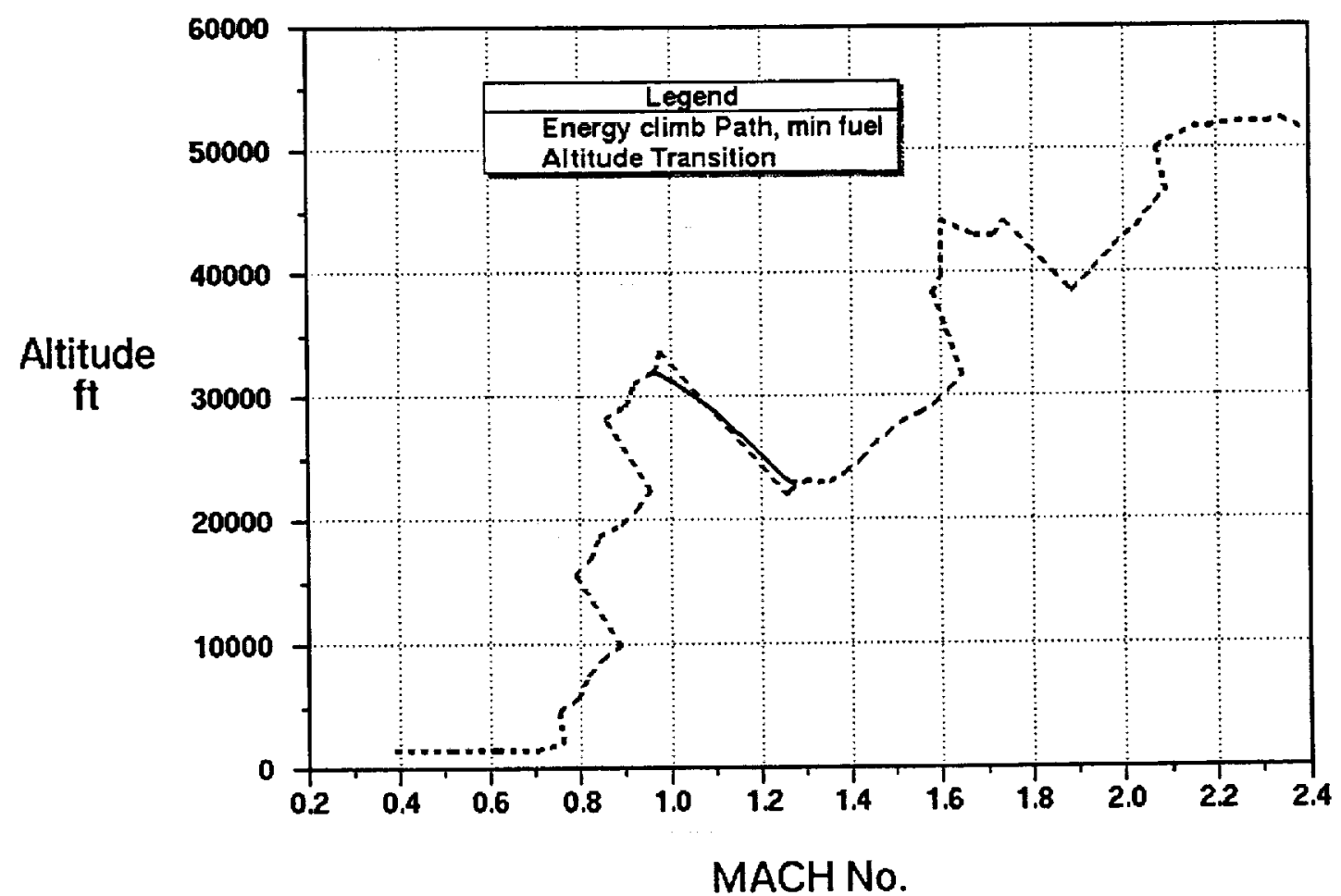

Figure 13. Transonic altitude transition for $N_{1}=0.97, N_{2}=1.05$, nonlinear determination of $\bar{v}$ and $\bar{\gamma}$. 


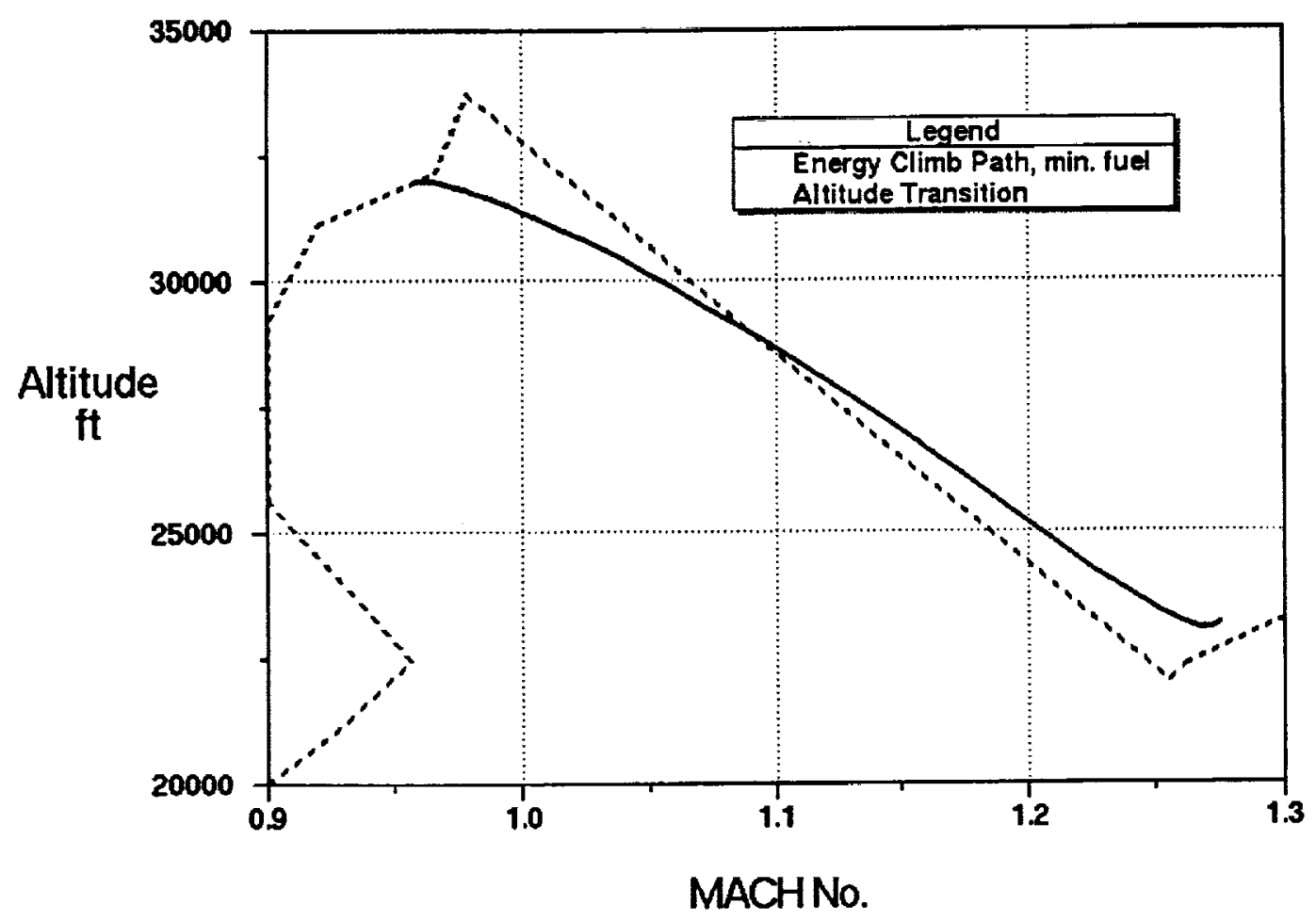

Figure 14. Altitude transition in the transonic region.

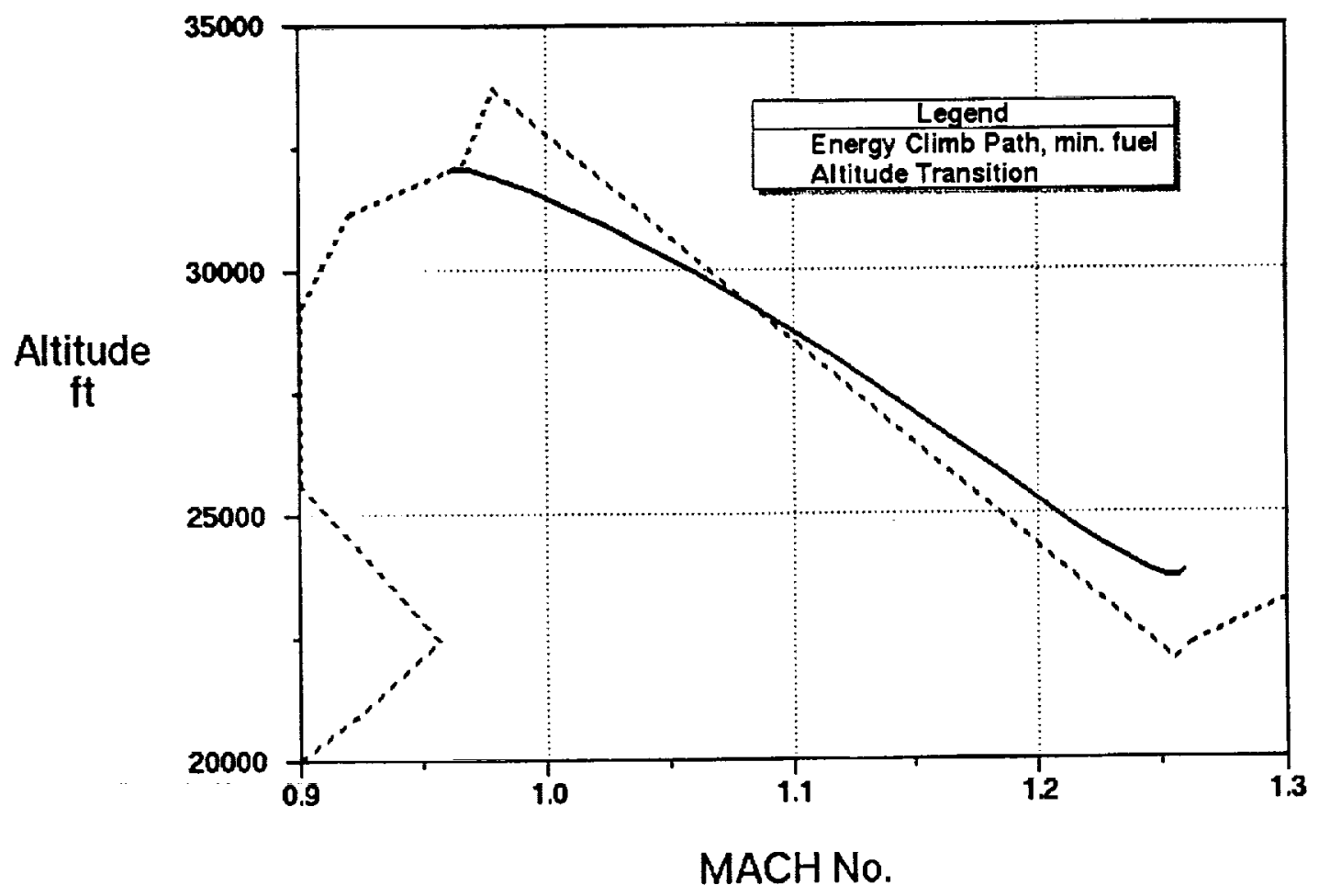

Figure 15. Altitude transition, linear determination of $\bar{v}$ and $\bar{\gamma}$. 


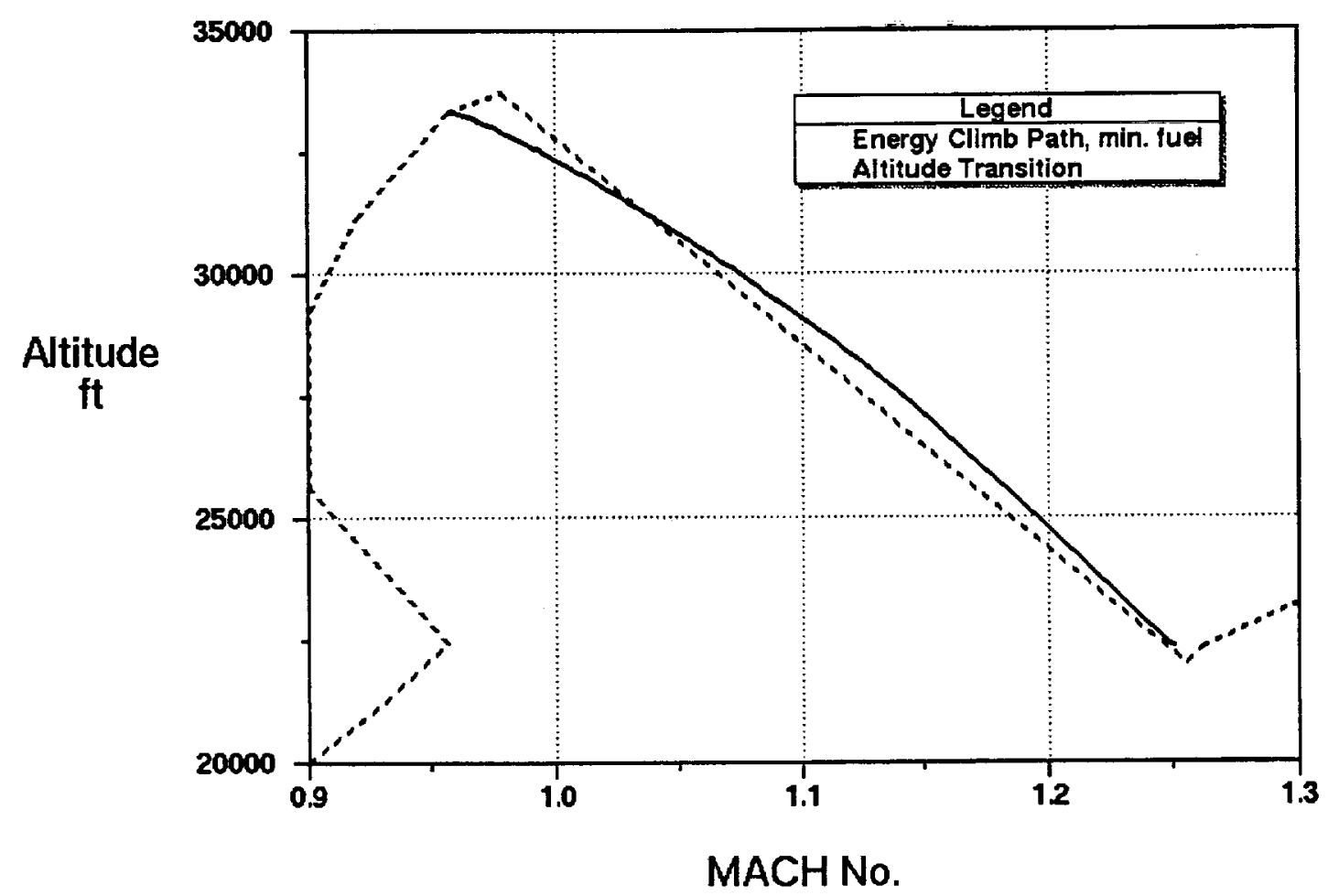

Figure 16. Altitude transition for $N_{1}=0.5, N_{2}=1.5$.

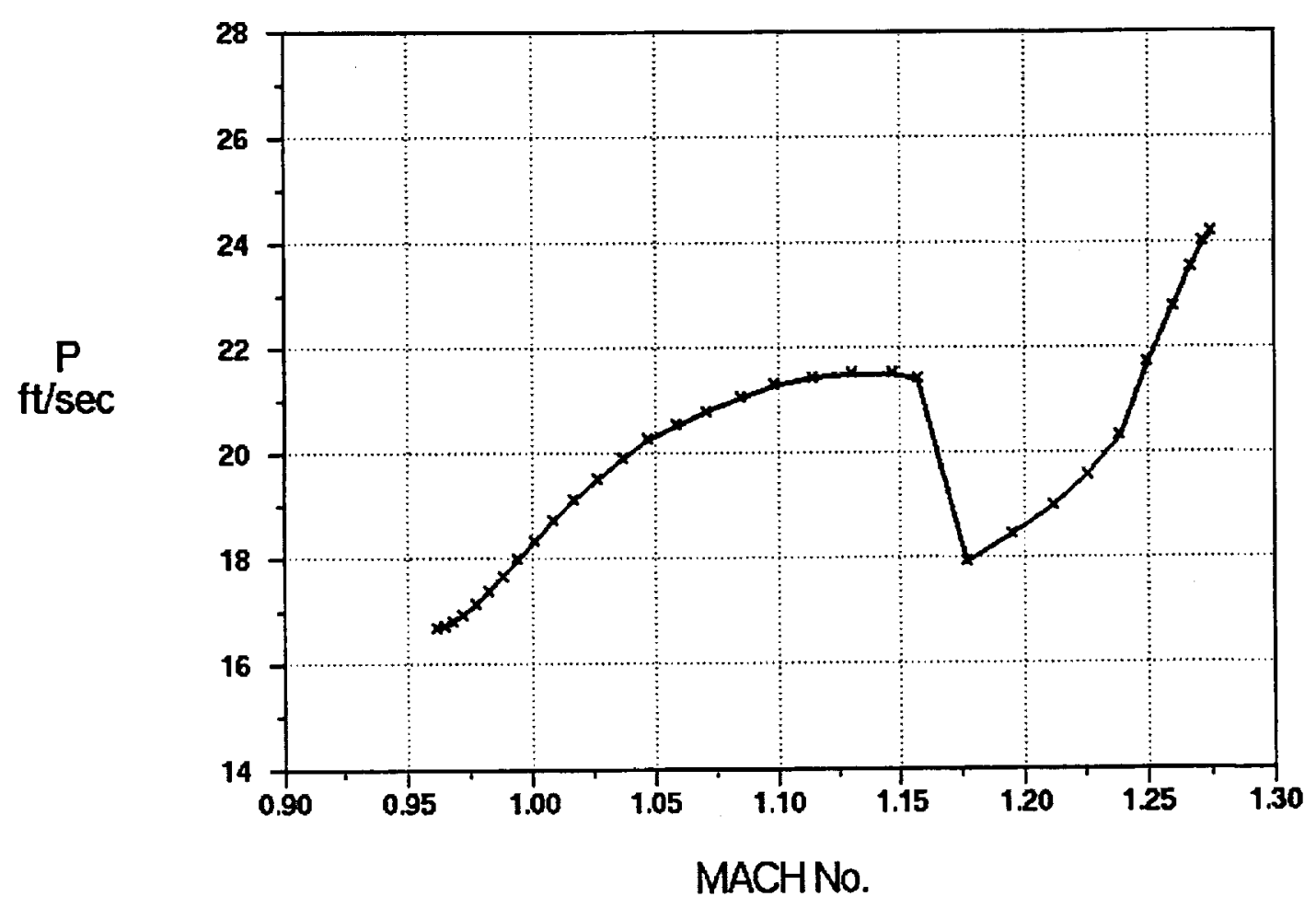

Figure 17. Energy rate during altitude transition, $N_{1}=0.97, N_{2}=1.05$. 


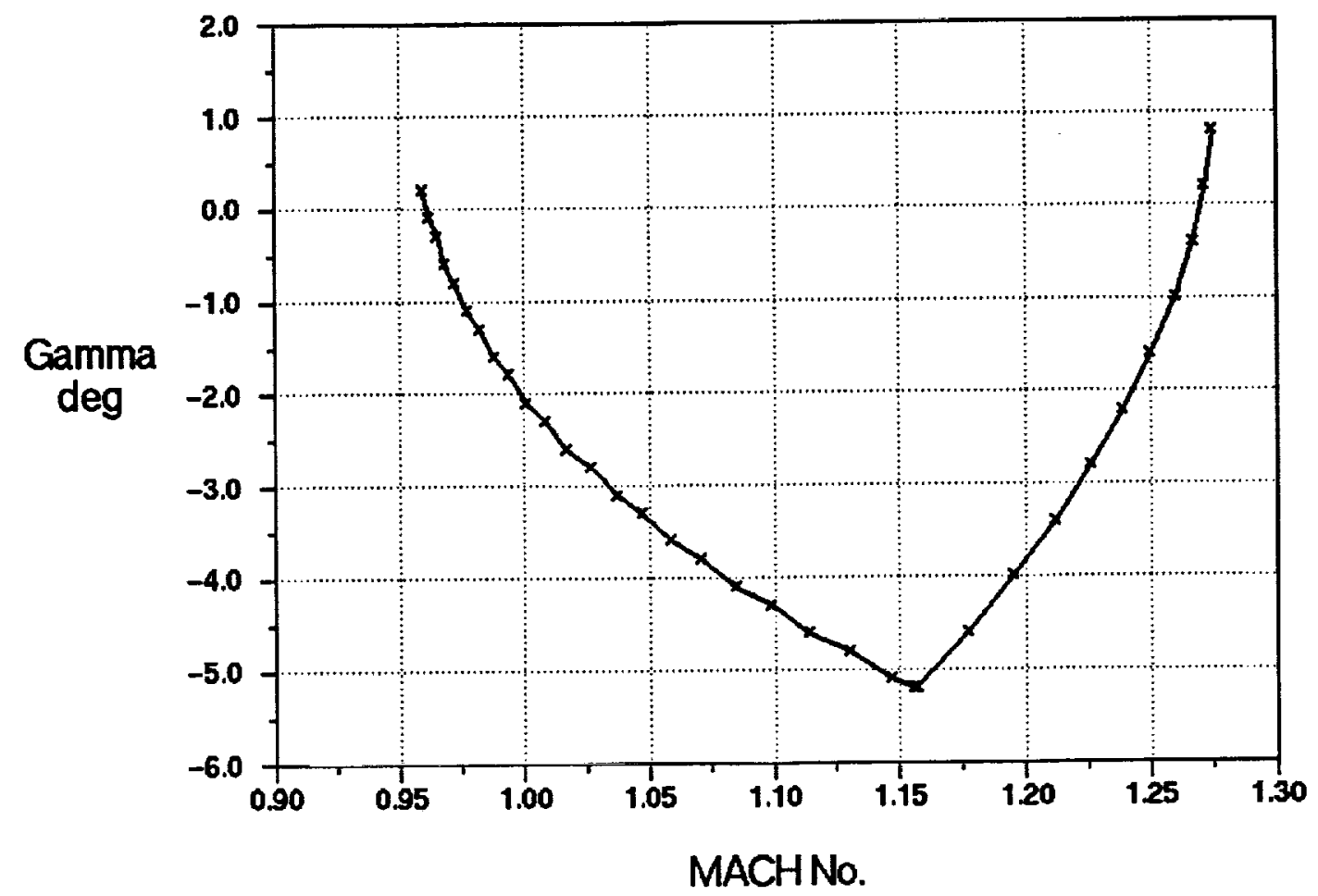

Figure 18. Flight path angle during altitude transition, $N_{1}=0.97, N_{2}=1.05$.

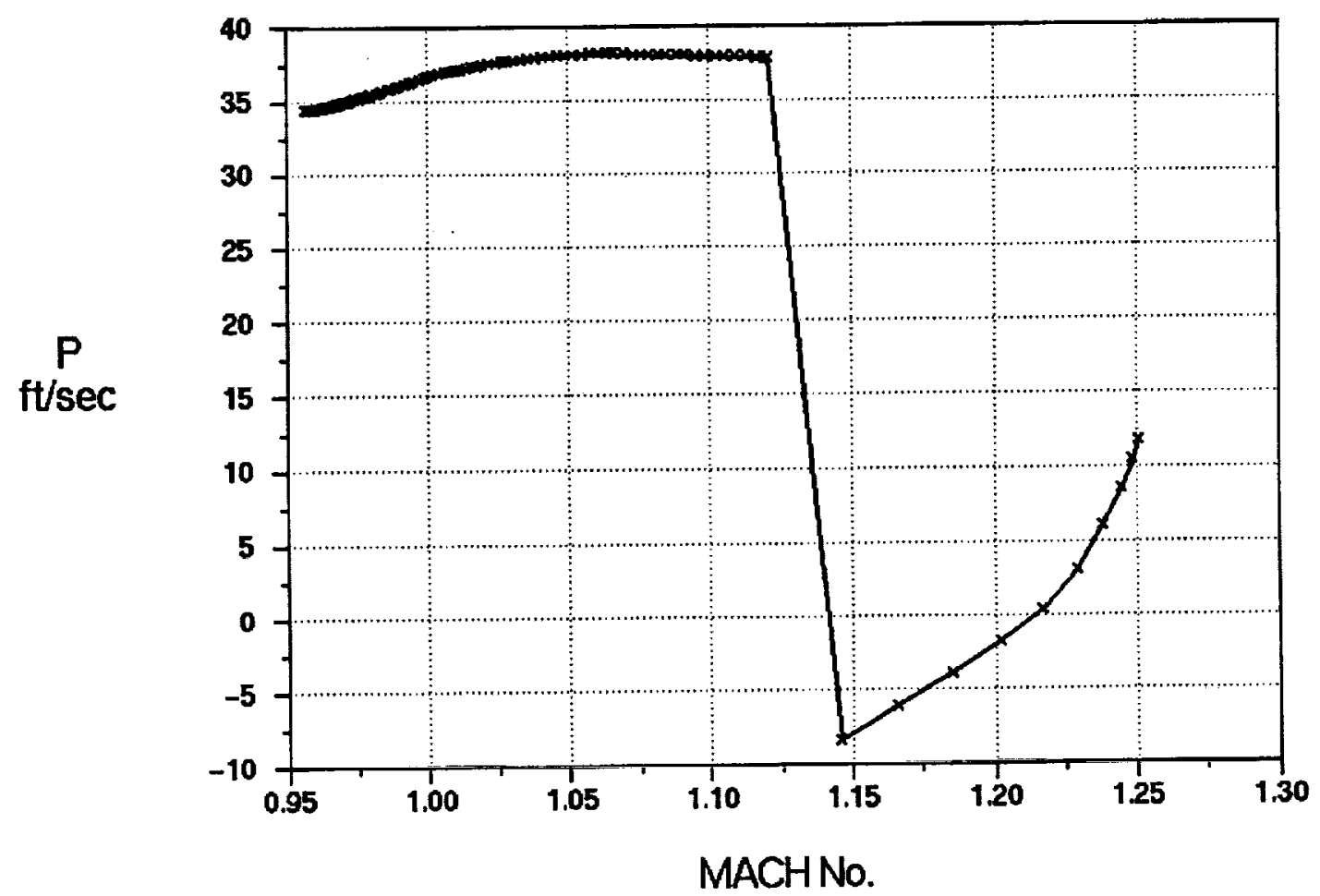

Figure 19. Energy rate during altitude transition, $N_{1}=0.5, N_{2}=1.5$. 


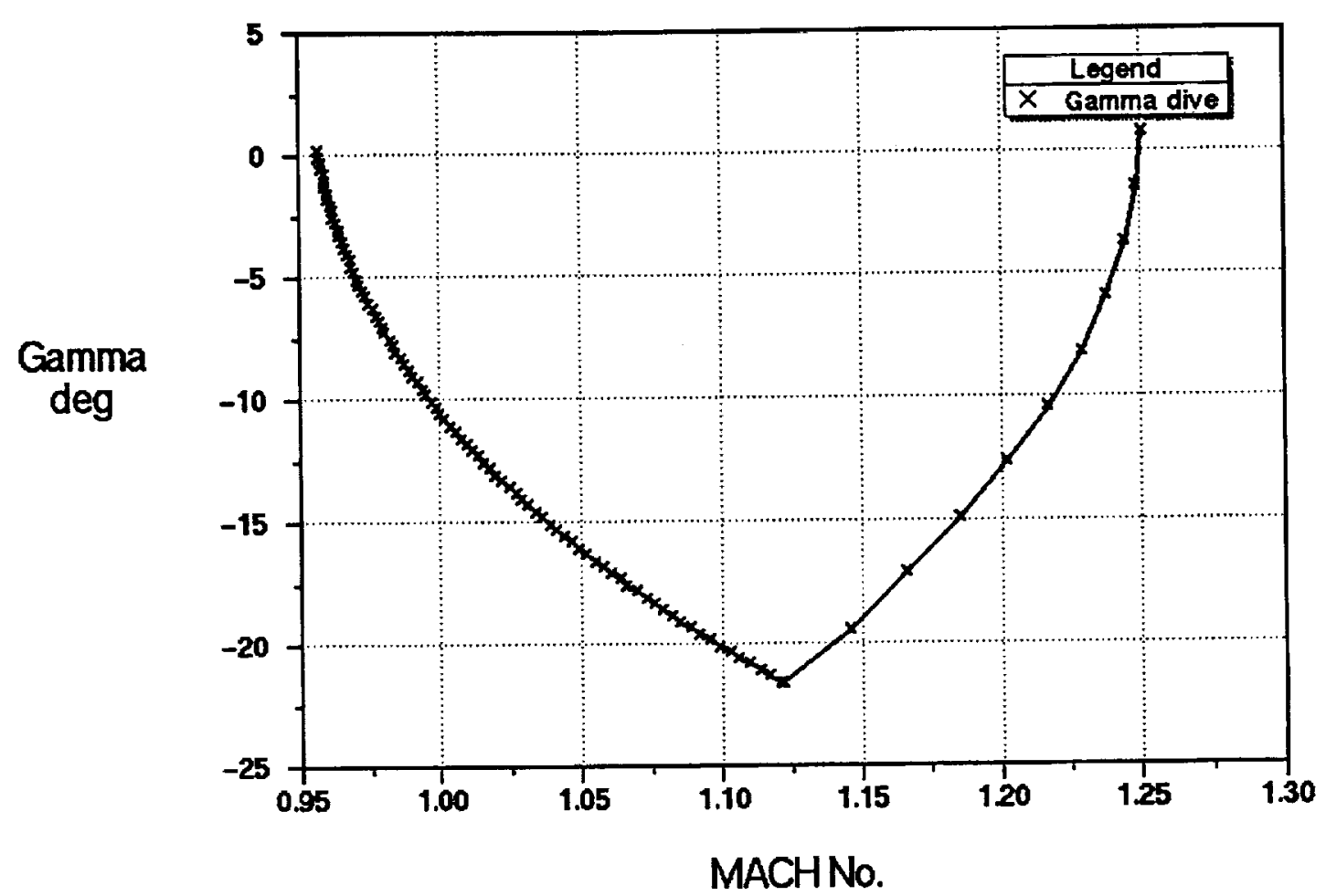

Figure 20. Flight path angle during altitude transition, $N_{1}=0.5, N_{2}=1.5$. 
Public reporting burden for this collection of intormation is estimated to average i hour pep response, including the time for reviewing instructions, searching existing data sources, gathering and maintaining the data needed, and completing and reviewing the collection of information. Send comments regarding this burden estimate or any other aspect of this collection of information, including sugestions for reducing this burden, to Washington Headquarters Services, Directorate for information Operations and Reports, 1215 Jelferson Davis Highway, Suite 1204, Arlington, VA 22202-4302, and to the Office of Management and Budget, Paperwork Reduction Project (0704-0188), Washington, DC 20503.

\begin{tabular}{|l|l|l} 
1. AGENCY USE ONLY (Leave blank) & 2. REPORT DATE & 3. REPORT TYPE AND DATES COVERED
\end{tabular} March 1998 Technical Memorandum

4. TITLE AND SUBTITLE 5. FUNDING NUMBERS

Optimization of Supersonic Transport Trajectories RTOPO

$522-41-42$

6. AUTHOR(S)

Mark D. Ardema, * Robert Windhorst,* and James Phillips

8. PERFORMING ORGANIZATION REPORT NUMBER

Ames Research Center

Moffett Field, CA 94035-1000

A-98-09997

9. SPONSORING/MONITORING AGENCY NAME(S) AND ADDRESS(ES)

10. SPONSORING/MONITORING AGENCY REPORT NUMBER

National Aeronautics and Space Administration

Washington, DC 20546-0001

NASA/TM-1998-112223

11. SUPPLEMENTARY NOTES

Point of Contact: James Phillips, Ames Research Center, MS 237-11, Moffett Field, CA 94035-1000 (650) 604-5789

* Santa Clara University, Santa Clara, Califomia

12a. DISTRIBUTION/AVAILABILITY STATEMENT

12b. DISTRIBUTION CODE

Unclassified - Unlimited

Subject Category 08

13. ABSTRACT (Maximum 200 words)

This paper develops a near-optimal guidance law for generating minimum fuel, time, or cost fixed-range trajectories for supersonic transport aircraft. The approach uses a choice of new state variables along with singular perturbation techniques to time-scale decouple the dynamic equations into multiple equations of single order (second order for the fast dynamics). Application of the maximum principle to each of the decoupled equations, as opposed to application to the original coupled equations, avoids the two point boundary value problem and transforms the problem from one of a functional optimization to one of multiple function optimizations. It is shown that such an approach produces well known aircraft performance results such as minimizing the Brequet factor for minimum fuel consumption and the energy climb path. Furthermore, the new state variables produce a consistent calculation of flight path angle along the trajectory, eliminating one of the deficiencies in the traditional energy state approximation. In addition, jumps in the energy climb path are smoothed out by integration of the original dynamic equations at constant load factor. Numerical results performed for a supersonic transport design show that a pushover dive followed by a pullout at nominal load factors are sufficient maneuvers to smooth the jump.

\begin{tabular}{|c|c|c|c|}
\hline \multirow{2}{*}{\multicolumn{3}{|c|}{$\begin{array}{l}\text { 14. SUBJECT TERMS } \\
\text { Supersonic aircraft trajectories, Singular perturbations, } \\
\text { Transonic altitude discontinuity }\end{array}$}} & $\begin{array}{l}\text { 15. NUMBER OF PAGES } \\
57\end{array}$ \\
\hline & & & $\begin{array}{r}\text { 16. PRICE CODE } \\
\text { A04 }\end{array}$ \\
\hline $\begin{array}{l}\text { 17. SECURITY CLASSIFICATION } \\
\text { OF REPORT } \\
\text { Unclassified }\end{array}$ & $\begin{array}{l}\text { 18. SECURITY CLASSIFICATION } \\
\text { OF THIS PAGE } \\
\text { Unclassified }\end{array}$ & $\begin{array}{l}\text { 19. SECURITY CLASSIFICATION } \\
\text { OF ABSTRACT }\end{array}$ & 20. LIMITATION OF ABSTRACT \\
\hline
\end{tabular}

